\title{
Steiner Tree Approximation via Iterative Randomized Rounding ${ }^{1}$
}

\author{
JAROSŁAW BYRKA \\ EPFL, Lausanne, Switzerland and University of Wrocław, Poland \\ and \\ FABRIZIO GRANDONI \\ IDSIA, University of Lugano, Switzerland \\ and \\ THOMAS ROTHVOB \\ EPFL, Lausanne, Switzerland and M.I.T., Cambridge, USA \\ and \\ LAURA SANITÀ \\ EPFL, Lausanne, Switzerland and University of Waterloo, Canada
}

The Steiner tree problem is one of the most fundamental NP-hard problems: given a weighted undirected graph and a subset of terminal nodes, find a minimum-cost tree spanning the terminals. In a sequence of papers, the approximation ratio for this problem was improved from 2 to 1.55 [Robins,Zelikovsky-'05]. All these algorithms are purely combinatorial. A long-standing open problem is whether there is an LP relaxation of Steiner tree with integrality gap smaller than 2 [Vazirani,Rajagopalan-'99].

In this paper we present an LP-based approximation algorithm for Steiner tree with an improved approximation factor. Our algorithm is based on a, seemingly novel, iterative randomized rounding technique. We consider an LP relaxation of the problem, which is based on the notion of directed components. We sample one component with probability proportional to the value of the associated variable in a fractional solution: the sampled component is contracted and the LP is updated consequently. We iterate this process until all terminals are connected. Our algorithm delivers a solution of cost at most $\ln (4)+\varepsilon<1.39$ times the cost of an optimal Steiner tree. The algorithm can be derandomized using the method of limited independence.

As a byproduct of our analysis, we show that the integrality gap of our LP is at most 1.55, hence answering the mentioned open question.

Categories and Subject Descriptors: F.2.2 [Computations on discrete structures]: Nonnumerical Algorithms and Problems

General Terms: Algorithms, Theory

Additional Key Words and Phrases: Approximation algorithms, linear programming relaxations, network design, randomized algorithms

\section{INTRODUCTION}

Given an undirected $n$-node graph $G=(V, E)$, with edge costs (or weights) $c: E \rightarrow$ $\mathbb{Q}_{+}$, and a subset of nodes $R \subseteq V$ (terminals), the Steiner tree problem asks for a tree $S$ spanning the terminals, of minimum cost $c(S):=\sum_{e \in S} c(e)$. Note that $S$ might contain some other nodes, besides the terminals (Steiner nodes). Steiner tree is one of the classic and most fundamental problems in Computer Science and

\footnotetext{
${ }^{1}$ A preliminary version of this paper appeared in STOC'10 [Byrka et al. 2010]. Emails: J. Byrka jby@ii.uni.wroc.pl, F. Grandoni fabrizio@idsia.ch (partially supported by ERC Starting Grant NEWNET 279352), T. Rothvoß rothvoss@math.mit.edu, L. Sanità lsanita@uwaterloo.ca.
} 
Operations Research, with great theoretical and practical relevance. This problem emerges in a number of contexts, such as the design of VLSI, optical and wireless communication systems, as well as transportation and distribution networks (see, e.g., [Hwang et al. 1992]).

The Steiner tree problem appears already in the list of NP-hard problems in the book by Garey and Johnson [1979]. In fact, it is NP-hard to find solutions of cost less than $\frac{96}{95}$ times the optimal cost [Bern and Plassmann 1989; Chlebík and Chlebíková 2008]. Hence, the best one can hope for is an approximation algorithm with a small but constant approximation guarantee. Recall that an $\alpha$-approximation algorithm for a minimization problem is a polynomial time algorithm that produces solutions which are guaranteed to have cost at most $\alpha$ times the optimum value.

Without loss of generality, we can replace the input graph by its metric closure ${ }^{2}$. A terminal spanning tree is a Steiner tree without Steiner nodes: such a tree always exists in the metric closure of the graph. It is well-known that a minimum-cost terminal spanning tree is a 2-approximation for the Steiner tree problem [Gilbert and Pollak 1968; Vazirani 2001].

A sequence of improved approximation algorithms appeared in the literature [Karpinski and Zelikovsky 1997; Prömel and Steger 2000; Zelikovsky 1993], culminating with the famous $1+\frac{\ln (3)}{2}+\varepsilon<1.55$ approximation algorithm by Robins and Zelikovsky [2005]. (Here $\varepsilon>0$ is an arbitrarily small constant). All these improvements are based on the notion of $k$-restricted Steiner tree, which is defined as follows. A component is a tree whose leaves coincide with a subset of terminals. A $k$-restricted Steiner tree $S$ is a collection of components, with at most $k$ terminals each ( $k$-components), whose union induces a Steiner tree. The cost of $S$ is the total cost of its components, counting duplicated edges with their multiplicity (see [Borchers and Du 1997] for more details). The $k$-Steiner ratio $\rho_{k} \geq 1$ is the supremum of the ratio between the cost opt ${ }_{k}$ of the optimal $k$-restricted Steiner tree and the cost opt of the optimal (unrestricted) Steiner tree. The following result by Borchers and $\mathrm{Du}$ [1997] shows that, in order to have a good approximation, it is sufficient to consider $k$-restricted Steiner trees for a large enough, constant $k$.

ThEOREM 1. [Borchers and Du 1997] Let $r$ and $s$ be the non-negative integers satisfying $k=2^{r}+s$ and $s<2^{r}$. Then

$$
\rho_{k}=\frac{(r+1) 2^{r}+s}{r 2^{r}+s} \leq 1+\frac{1}{\left\lfloor\log _{2} k\right\rfloor} .
$$

The mentioned approximation algorithms exploit the notion of $k$-component within a local-search framework. They start with a minimum-cost terminal spanning tree (which is 2-approximate), and iteratively improve it. At each iteration, they add to the current solution a $k$-component, chosen according to some greedy strategy, and remove redundant edges. The process is iterated until no further improvement is achievable. Different algorithms use different greedy criteria.

Despite the efforts of many researchers in the last 10 years, the above framework did not provide any further improvement after [Robins and Zelikovsky 2000; 2005].

\footnotetext{
${ }^{2}$ The metric closure of a weighted graph is a complete weighted graph on the same node set, with weights given by shortest path distances with respect to original weights.

Journal of the ACM, Vol. ?, No. ?, ? 20?
} 
This motivated our search for alternative methods. One standard approach is to exploit a proper LP relaxation (see, e.g., [Goemans and Myung 1993] for a list of LP relaxations of Steiner tree). A natural formulation for the problem is the undirected cut formulation (see [Goemans and Williamson 1995; Vazirani 2001]). Here we have a variable for each edge of the graph and a constraint for each cut separating the set of terminals. Each constraint forces a solution to pick at least one edge crossing the corresponding cut. Considering the LP relaxation of this formulation, 2-approximation algorithms can be obtained either using primal-dual schemes (see [Goemans and Williamson 1995]) or iterative rounding (see [Jain 2001]). However, this relaxation has an integrality gap of 2 already in the spanning tree case, i.e., when $R=V$ (see example 22.10 in [Vazirani 2001]).

Another well-studied, but more promising, LP is the bidirected cut relaxation [Chakrabarty et al. 2008; Edmonds 1967; Rajagopalan and Vazirani 1999]. Let us fix an arbitrary terminal $r$ (root). Replace each edge $\{u, v\}$ by two directed edges $(u, v)$ and $(v, u)$ of cost $c(\{u, v\})$. For a given cut $U \subseteq V$, define $\delta^{+}(U)=\{(u, v) \in$ $E \mid u \in U, v \notin U\}$ as the set of edges leaving $U$. The mentioned relaxation is

$$
\begin{array}{rr}
\min \sum_{e \in E} c(e) z_{e} & (\mathrm{BCR}) \\
\text { s.t. } \sum_{e \in \delta^{+}(U)} z_{e} \geq 1, & \forall U \subseteq V \backslash\{r\}: U \cap R \neq \emptyset ; \\
z_{e} \geq 0, & \forall e \in E .
\end{array}
$$

We can consider the value $z_{e}$ as the capacity which we are going to install on the directed edge $e$. The LP can then be interpreted as computing the minimum-cost capacities that support a flow of 1 from each terminal to the root. In a seminal work, Edmonds [1967] showed that BCR is integral in the spanning tree case.

Theorem 2. [Edmonds 1967] For $R=V$, the polyhedron of BCR is integral.

The best-known lower bound on the integrality gap of BCR is $8 / 7$ [Könemann et al. 2011; Vazirani 2001]. The best-known upper bound is 2, though BCR is believed to have a smaller integrality gap than the undirected cut relaxation [Rajagopalan and Vazirani 1999]. Chakrabarty et al. [2008] report that the structure of the dual to BCR is highly asymmetric, which complicates a primal-dual approach. Moreover, iterative rounding based on picking a single edge cannot yield good approximations, as was pointed out in [Rajagopalan and Vazirani 1999].

Finding a better-than-2 LP relaxation of the Steiner tree problem is a longstanding open problem [Chakrabarty et al. 2008; Rajagopalan and Vazirani 1999]. We remark that good LP-bounds, besides potentially leading to better approximation algorithms for Steiner tree, might have a much wider impact. This is because Steiner tree appears as a building block in several other problems, and the best approximation algorithms for some of those problems are LP-based. Strong LPs are also important in the design of (practically) efficient and accurate heuristics.

\subsection{Our Results and Techniques}

The mayor contribution of this paper lies in introducing novel linear programming techniques for the Steiner tree problem, along with a continuous sampling and 


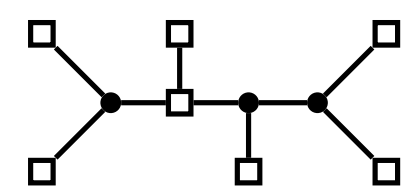

(a)

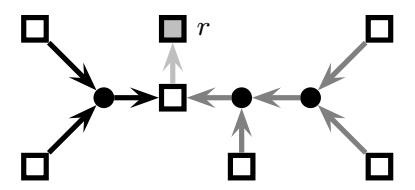

(b)

Fig. 1. (a) A Steiner tree $S$, where rectangles denote terminals and circles represent Steiner nodes. (b) Edges of $S$ are directed towards a root $r$. The directed components of $S$ are depicted with different colors.

re-optimization approach that appears to be new in the field of approximation algorithms in general. The main result of this paper is as follows.

Theorem 3. For any constant $\varepsilon>0$, there is a polynomial-time $(\ln (4)+\varepsilon)$ approximation algorithm for the Steiner tree problem.

This can be improved to $73 / 60+\varepsilon$ in the well-studied special case of quasi-bipartite graphs (where non-terminal nodes are pairwise not adjacent).

Our algorithm is based on the following directed-component cut relaxation for the Steiner tree problem (a similar relaxation is considered in [Polzin and VahdatiDaneshmand 2003]). Consider any subset of terminals $R^{\prime} \subseteq R$, and any $r^{\prime} \in R^{\prime}$. Let $C$ be the minimum-cost Steiner tree on terminals $R^{\prime}$, with edges directed towards $r^{\prime}$ (directed component). For a given directed component $C$, we let $c(C)$ be its cost, and $\operatorname{sink}(C)$ be its unique sink terminal (i.e. the only terminal that can be reached from all other terminals in $C$ ). We call the remaining terminals $\operatorname{sources}(C):=$ $V(C) \cap R \backslash\{\operatorname{sink}(C)\}$. The set of components obtained this way is denoted by $\mathbf{C}_{n}$. We say that a directed component $C \in \mathbf{C}_{n}$ crosses a set $U \subseteq R$ if $C$ has at least one source in $U$ and the sink outside. By $\delta_{\mathbf{C}_{n}}^{+}(U)$ we denote the set of directed components crossing $U$. Furthermore, we choose an arbitrary terminal $r$ as a root. Our LP relaxation is then:

$$
\begin{array}{rrr}
\min \sum_{C \in \mathbf{C}_{n}} c(C) x_{C} & (\mathrm{DCR}) & \\
\text { s.t. } \sum_{C \in \delta_{\mathbf{C}_{n}}^{+}(U)} x_{C} \geq 1, & \forall U \subseteq R \backslash\{r\}, U \neq \emptyset ; \\
x_{C} \geq 0, & \forall C \in \mathbf{C}_{n} .
\end{array}
$$

DCR is trivially a relaxation of the Steiner tree problem. In fact, one can direct the edges of the optimal Steiner tree $S^{*}$ towards terminal $r$, and split the edge set of $S^{*}$ at interior terminals. This yields a set of directed components $\mathbf{C} \subseteq \mathbf{C}_{n}$ (see Figure 1). Observe that any $C \in \mathbf{C}$ must be an optimal Steiner tree on terminals $R \cap V(C)$. Consequently, setting $x_{C}=1$ for any $C \in \mathbf{C}$, and the remaining variables to zero, provides a feasible solution to DCR of cost $\sum_{C \in \mathbf{C}} c(C)=c\left(S^{*}\right)=$ opt.

Unfortunately the cardinality of $\mathbf{C}_{n}$ is exponential. However, we will see that, for any constant $\varepsilon>0$, one can compute a $(1+\varepsilon)$-approximate fractional solution to DCR in polynomial time. This is achieved by restricting $\mathbf{C}_{n}$ to the directed components $\mathbf{C}_{k}$ that contain at most a (big) constant number $k$ of terminals (directed

Journal of the ACM, Vol. ?, No. ?, ? 20?. 
k-components).

We combine our LP with a (to the best of our knowledge) novel iterative randomized rounding technique. We solve the LP (approximately), sample one component $C$ with probability proportional to its value $x_{C}$ in the near-optimal fractional solution $x$, contract $C$ into its sink node $\operatorname{sink}(C)$, and reoptimize the LP. We iterate this process until only one terminal remains, i.e., until all terminals are connected by the sampled components. A fairly simple analysis provides a $3 / 2+\varepsilon$ bound on the approximation ratio. With a refined analysis, we improve this bound to $\ln (4)+\varepsilon$. Our algorithm can be derandomized.

We remark that our algorithm combines features of randomized rounding (where typically variables are rounded randomly, but simultaneously) and iterative rounding (where variables are rounded iteratively, but deterministically). We believe that our iterative randomized rounding technique will also find other applications, and is henceforth of independent interest.

The key insight in our analysis is to quantify the expected reduction of the cost of the optimal Steiner tree in each iteration. To show this, we exploit a novel Bridge Lemma, relating the cost of terminal spanning trees with the cost of fractional solutions to DCR. The proof of the lemma is based on Theorem 2 [Edmonds 1967]. In our opinion, our analysis of the reduction in the cost of the optimal Steiner tree in each iteration using the Bridge Lemma is simpler (or at least more intuitive) than the analogous results in [Robins and Zelikovsky 2005].

As an easy consequence of our analysis, we obtain that the integrality gap of DCR is at most $1+\ln (2)<1.694$, hence answering the mentioned open problem in [Chakrabarty et al. 2008; Rajagopalan and Vazirani 1999]. Combining our Bridge Lemma with the algorithm and analysis by Robins and Zelikovsky [2005], we obtain the following improved result.

TheOREm 4. For any $\varepsilon>0$, there is an algorithm for the Steiner tree problem which computes a solution of cost at most $1+\frac{\ln (3)}{2}+\varepsilon$ times the cost of the optimal fractional solution to DCR. The running time of the algorithm is polynomial for constant $\varepsilon$.

The above theorem immediately implies a $1+\ln (3) / 2<1.55$ upper bound on the integrality gap of DCR, by letting $\varepsilon$ tend to zero (the running time is irrelevant with that respect). As mentioned before, integrality gap results of this type often provide new insights into variants and generalizations of the original problem. We expect that this will be the case with the above theorem as well, since Steiner tree appears as a building block in many other problems.

We also show that the integrality gap of DCR and BCR are at least 8/7 $>1.142$ and $36 / 31>1.161$, respectively.

\subsection{Related Work}

A sign of importance of the Steiner tree problem is that it appears either as a subproblem or as a special case of many other problems in network design. A (certainly incomplete) list contains Steiner forest [Agrawal et al. 1995; Goemans and Williamson 1995], prize-collecting Steiner tree [Archer et al. 2009; Goemans and Williamson 1995], virtual private network [Eisenbrand and Grandoni 2005; Eisenbrand et al. 2007; Grandoni, Rothvoß, and Sanità 2011; Gupta et al. 2001], 
single-sink rent-or-buy [Eisenbrand et al. 2010; Grandoni, Rothvoß, and Sanità 2011; Gupta et al. 2007; Jothi and Raghavachari 2009], connected facility location [Eisenbrand et al. 2010; Grandoni and Rothvoß 2011; Swamy and Kumar 2004], and single-sink buy-at-bulk [Grandoni and Italiano 2006; Gupta et al. 2007; Grandoni, Rothvoß, and Sanità 2011; Talwar 2002].

Both the previously cited primal-dual and iterative rounding approximation techniques apply to a more general class of problems. In particular, the iterative rounding technique introduced by Jain [2001] provides a 2-approximation for the Steiner network problem, and the primal-dual framework developed by Goemans and Williamson [1995] gives the same approximation factor for a large class of constrained forest problems.

Regarding the integrality gap of LP relaxations of the Steiner tree problem, upper bounds better than 2 are known only for special graph classes. For example, BCR has an integrality gap smaller than 2 on quasi-bipartite graphs, where non-terminal nodes induce an independent set. For such graphs Rajagopalan and Vazirani [1999] (see also [Rizzi 2003]) gave an upper bound of $3 / 2$ on the gap. This was recently improved to $4 / 3$ by Chakrabarty, Devanur and Vazirani [2008]. Still, for this class of graphs the lower bound of $8 / 7$ holds [Könemann et al. 2011; Vazirani 2001]. Könemann, Pritchard and Tan [2011] showed that for a different LP formulation, which is stronger than BCR, the integrality gap is upper-bounded by $\frac{2 b+1}{b+1}$, where $b$ is the maximum number of Steiner nodes in full components. All the mentioned LPs can be solved in polynomial time, while we solve DCR only approximately: from a technical point of view, we indeed solve exactly a relaxation of the $k$-restricted Steiner tree problem. Under additional constraints, Steiner tree admits better approximations. In particular, a PTAS can be obtained by the technique of Arora [1998] if the nodes are points in a fixed-dimension Euclidean space, and using the algorithm of Borradaile, Kenyon-Mathieu and Klein [2009] for planar graphs.

After the circulation of a preliminary version of our results, Chakrabarty, Könemann, and Pritchard [Chakrabarty et al. 2010a] proved an integrality gap of $\sqrt{3}<1.74$ for a different (but equivalent) LP-relaxation, using a different approach.

\subsection{Organization}

The rest of this paper is organized as follows. In Section 2 we give some definitions and basic results. In Section 3 we show how to approximate DCR and prove our Bridge Lemma. In Section 4 we present a simple expected $(1.5+\varepsilon)$-approximation for the problem. This result is improved to $\ln (4)+\varepsilon$ in Section 5. The special case of quasi-bipartite graphs is considered in Section 5.1. We derandomize our algorithm in Section 6. Finally, in Section 7 we discuss the integrality gap of DCR, and compare DCR with BCR.

\section{PRELIMINARIES}

We use Opt to denote the optimal integral solution, and opt $=c(\mathrm{Opt})$. The cost of an optimal solution to DCR (for the input instance) is termed $\mathrm{opt}_{f}$. We will consider algorithms consisting of a sequence of iterations, each one considering different subproblems. We will use superscript to denote the considered iteration

$t$. For example, $\operatorname{opt}_{f}^{t}$ denotes the cost of an optimal fractional solution at the beginning of iteration $t$.

Journal of the ACM, Vol. ?, No. ?, ? 20?. 
For a given (directed or undirected) component $C, R(C):=R \cap V(C)$ is the set of its terminals. Recall that DCR has an exponential number of variables and constraints. For this reason, our algorithms will consider approximate solutions to DCR with a polynomial-size support. Therefore, it is notationally convenient to represent a solution to DCR as a pair $(x, \mathbf{C})$, where $\mathbf{C} \subseteq \mathbf{C}_{n}$ is a subset of directed components and $x=\left\{x_{C}\right\}_{C \in \mathbf{C}}$ denotes the values that are associated to each such component. (Other variables are assumed to have value zero).

Let $T$ be a minimum-cost terminal spanning tree. It is a well-known fact that $c(T) \leq 2$. opt (see e.g. Theorem 3.3 in [Vazirani 2001]). Extending the standard proof, this bound also holds w.r.t. our LP relaxation.

Lemma 5. $c(T) \leq 2 \cdot \mathrm{opt}_{f}$.

Proof. Let $(x, \mathbf{C})$ be an optimal fractional solution to DCR. For each component $C \in \mathbf{C}$, obtain an undirected TSP tour on $R(C)$ of cost at most $2 c(C)$, remove one edge of the tour, and direct the remaining edges towards $\operatorname{sink}(C)$. Install capacity $x_{C}$ cumulatively on the directed edges of the resulting arborescence. This induces a fractional solution to DCR of cost at most $2 \cdot \mathrm{opt}_{f}$, with the property that only components with 2 terminals and without Steiner nodes are used. This also provides a feasible fractional solution to BCR of the same cost. Since BCR without Steiner nodes is integral by Theorem 2, the claim follows.

Let $R^{\prime}$ be a subset of $k$ terminals. Consider a given Steiner tree $S$, with edge weights $c$, containing the terminals $R^{\prime}$. The weight function $c$ associated to $S$, if not specified, will be clear from the context. Let us collapse the terminals $R^{\prime}$ into one node, and call $G^{\prime}$ the resulting (possibly, multi-)graph. Let $S^{\prime} \subseteq S$ be a minimum spanning tree of $G^{\prime}$. Observe that $S^{\prime}$ will contain all the edges of $S$ but $k-1$ edges, since collapsing $R^{\prime}$ decreases the number of nodes in $S$ by $k-1$. We call the latter edges the bridges of $S$ w.r.t. $R^{\prime}$, and denote them by $\operatorname{Br}_{S}\left(R^{\prime}\right)^{3}$. Intuitively, if we imagine to add zero cost dummy edges between the terminals $R^{\prime}, \operatorname{Br}_{S}\left(R^{\prime}\right)$ is a maximum-cost subset of edges that we could remove from $S$ and still have a connected spanning subgraph. In other terms,

$$
\operatorname{Br}_{S}\left(R^{\prime}\right)=\operatorname{argmax}\left\{c(B) \mid B \subseteq S, S \backslash B \cup\left(\begin{array}{c}
R^{\prime} \\
2
\end{array}\right) \text { connects } V(S)\right\} .
$$

Let us abbreviate $\operatorname{br}_{S}\left(R^{\prime}\right):=c\left(\operatorname{Br}_{S}\left(R^{\prime}\right)\right)$. For a (directed or undirected) component $C^{\prime}$, we use $\operatorname{Br}_{S}\left(C^{\prime}\right)$ and $\operatorname{br}_{S}\left(C^{\prime}\right)$ as shortcuts for $\operatorname{Br}_{S}\left(R\left(C^{\prime}\right)\right)$ and $\operatorname{br}_{S}\left(R\left(C^{\prime}\right)\right)$, respectively.

In the analysis, it is often convenient to turn a given Steiner tree $S$ into a rooted, possibly non-complete, binary tree as follows (see also [Karpinski and Zelikovsky 1997]). First, we append a dummy node $v^{\prime}$ to each non-leaf terminal $v$ with a dummy edge of cost zero, and we replace $v$ with $v^{\prime}$ in the set of terminals. Note that now terminals coincide with the leaves of the tree. Second, for any internal node $v$ of degree $d \geq 4$, we replace $v$ with a path $v_{1}, \ldots, v_{d-2}$ of dummy nodes and dummy edges of cost zero. Node $v_{1}$ and $v_{d-2}$ inherit two neighbors of $v$ and all the other $v_{i}$ one neighbor of $v$, so that each original neighbor of $v$ is adjacent to exactly one node $v_{i}$. Third, we contract nodes of degree 2. Finally, we split an arbitrary

\footnotetext{
${ }^{3}$ As usual, we break ties according to edge indexes.
} 
edge by introducing a dummy root node $v$. We assign the cost of the original edge to one of the two resulting edges, and weight zero to the other edge. It is not hard to show that the resulting tree has height at most $|R|-1$. Given this reduction, it is easy to prove the following standard result.

Lemma 6. For any Steiner tree $S$ on terminals $R, \operatorname{br}_{S}(R) \geq \frac{1}{2} c(S)$.

Proof. Turn $S$ into a rooted binary tree as described above. For each Steiner node of $S$, mark the most expensive edge of the two edges going to its children. Let $B \subseteq S$ be the set of marked edges. Observe that $c(B) \geq \frac{1}{2} c(S)$. Furthermore, after contracting $R$, one can remove $B$ while keeping $S$ connected. (This is because there exists a path not intersecting $B$ from each internal node to some leaf). From the definition of bridges it follows that $\operatorname{br}_{S}(R) \geq c(B) \geq \frac{1}{2} c(S)$.

Throughout this paper, we sometimes identify a subgraph $G^{\prime}$ with its set of edges $E\left(G^{\prime}\right)$.

\section{A DIRECTED-COMPONENT CUT RELAXATION}

In this section we show how to solve DCR approximately (Section 3.1), and prove our Bridge Lemma (Section 3.2).

\subsection{Approximating DCR}

We next show how to compute a $(1+\varepsilon)$-approximate solution to DCR, for any given constant $\varepsilon>0$, in polynomial time. This is achieved in two steps. First of all, we introduce a relaxation $k$-DCR of the $k$-restricted Steiner tree problem. This relaxation can be solved exactly in polynomial time for any constant value of the parameter $k$ (Lemma 8). Then we show that the optimal solutions to $k$-DCR and DCR are close for large-enough $k$ (Lemma 7).

Let $\mathbf{C}_{k} \subseteq \mathbf{C}_{n}$ denote the set of directed components with at most $k$ terminals, and let $\delta_{\mathbf{C}_{k}}^{+}(U):=\delta_{\mathbf{C}_{n}}^{+}(U) \cap \mathbf{C}_{k}$. By the same arguments as for the unrestricted case, the following is a relaxation of the $k$-restricted Steiner tree problem:

$$
\begin{array}{lr}
\min \sum_{C \in \mathbf{C}_{k}} c(C) x_{C} & (k \text {-DCR }) \\
\text { s.t. } \sum_{C \in \delta_{\mathbf{C}_{k}}^{+}(U)} x_{C} \geq 1, \forall U \subseteq R \backslash\{r\}, U \neq \emptyset ; \\
x_{C} \geq 0, & \forall C \in \mathbf{C}_{k} .
\end{array}
$$

Let $\operatorname{opt}_{f, k}$ be the value of the optimal fractional solution to $k$-DCR. Trivially, $\operatorname{opt}_{f, k} \geq \mathrm{opt}_{f}$ since any feasible solution to $k$-DCR is also feasible for DCR. We can exploit the result by Borchers and $\mathrm{Du}$ [1997] to show that opt ${ }_{f, k}$ is indeed close to $\mathrm{opt}_{f}$ for large $k$.

Lemma 7. $\operatorname{opt}_{f, k} \leq \rho_{k} \cdot \mathrm{opt}_{f}$.

Proof. Let $(x, \mathbf{C})$ be an optimal fractional solution for DCR. We show how to construct a solution $\left(x^{\prime}, \mathbf{C}^{\prime}\right)$ to $k$-DCR with the claimed property. For any component $C \in \mathbf{C}$, we can apply Theorem 1 to obtain a list of undirected components $C_{1}, \ldots, C_{\ell}$ such that: (a) $\bigcup_{i=1}^{\ell} C_{i}$ connects the terminals in $C$, (b) any $C_{i}$ contains at most $k$ terminals, and (c) $\sum_{i=1}^{\ell} c\left(C_{i}\right) \leq \rho_{k} \cdot c(C)$. Next, we direct the edges of Journal of the ACM, Vol. ?, No. ?, ? 20?. 
all $C_{i}$ 's consistently towards $\operatorname{sink}(C)$ and increase the value of $x_{C_{i}}^{\prime}$ by $x_{C}$ for each $C_{i}$. The resulting solution $\left(x^{\prime}, \mathbf{C}^{\prime}\right)$ satisfies the claim.

It remains to solve $k$-DCR for $k=O(1)$. For any fixed $k$, in polynomial time one can consider any subset $R^{\prime} \subseteq R$ of at most $k$ terminals, and compute an optimal Steiner tree $Z$ on $R^{\prime 4}$. By considering each $r^{\prime} \in R^{\prime}$, and directing the edges of $Z$ towards $r^{\prime}$, one obtains all the directed components on terminals $R^{\prime}$. Consequently, $\left|\mathbf{C}_{k}\right|=O\left(k n^{k}\right)$ and the $k$-components can be listed in polynomial time.

LEMma 8. The optimal solution to $k-D C R$ can be computed in polynomial time for any constant $k$.

Proof. We define a directed auxiliary graph $G^{\prime}=\left(V^{\prime}, E^{\prime}\right)$, on node set $V^{\prime}=R \cup$ $\left\{v_{C} \mid C \in \mathbf{C}_{k}\right\}$. For every component $C$, insert edges $\left(u, v_{C}\right)$ for any $u \in \operatorname{sources}(C)$, and one edge $e_{C}=\left(v_{C}, \operatorname{sink}(C)\right)$. We observe that $k$-DCR is equivalent to a nonsimultaneous multicommodity flow problem, where any terminal in $R$ sends one unit of flow to the root and edges $e_{C}$ have $\operatorname{cost} c(C)$.

More precisely $k$-DCR is equivalent to the following compact LP:

$$
\begin{aligned}
& \min \sum_{C \in \mathbf{C}_{k}} c(C) x_{C} \\
& \text { s.t. } \sum_{e \in \delta^{+}(v)} f_{s}(e)-\sum_{e \in \delta^{-}(v)} f_{s}(e)=\left\{\begin{array}{ll}
1 & \text { if } v=s ; \\
-1 & \text { if } v=r ; \\
0 & \text { if } v \in V \backslash\{r, s\}, \\
f_{s}\left(e_{C}\right) & \leq x_{C},
\end{array} \quad \forall s \in R \backslash\{r\} ; \quad \forall s \in R \backslash\{r\}, C \in \mathbf{C}_{k} ;\right. \\
& f_{s}(e), x_{C} \geq 0,
\end{aligned} \quad \forall s \in R \backslash\{r\}, e \in E^{\prime}, C \in \mathbf{C}_{k} .
$$

Here $f_{s}(e)$ denotes the flow that terminal $s$ sends across edge $e$ and the capacity on edge $e_{C}$ is $x_{C}=\max _{s \in R \backslash\{r\}} f_{s}\left(e_{C}\right)$. An optimal solution of the latter LP can be computed in polynomial time, see e.g. [Khachiyan 1979; Grötschel et al. 1981] .

Putting everything together, we obtain the desired approximate solution to DCR.

Lemma 9. For any fixed $\varepsilon>0, a(1+\varepsilon)$-approximate solution $(x, \mathbf{C})$ to $D C R$ can be computed in polynomial time.

Proof. It is sufficient to solve $k$-DCR for $k:=2^{\lceil 1 / \varepsilon\rceil}$ with the algorithm from Lemma 8. Observe that $\rho_{k} \leq 1+\varepsilon$ (see again Theorem 1). The claim follows from Lemma 7.

\subsection{The Bridge Lemma}

We next prove our Bridge Lemma, which is the heart of our analysis. This lemma relates the cost of any terminal spanning tree to the cost of any fractional solution to DCR via the notion of bridges, and its proof is based on Edmonds' Theorem (Theorem 2).

\footnotetext{
${ }^{4}$ We recall that, given $k$ terminals, the dynamic-programming algorithm by Dreyfus and Wagner [1972] computes an optimal Steiner tree among them in $O\left(3^{k} n+2^{k} n^{2}+n^{3}\right)$ worst-case time. A faster parameterized algorithm can be found in [Mölle et al. 2006].

${ }^{5}$ Note that this LP can even be solved in strongly-polynomial time using the Frank-Tardos algorithm [Frank and Tardos 1987]
} 


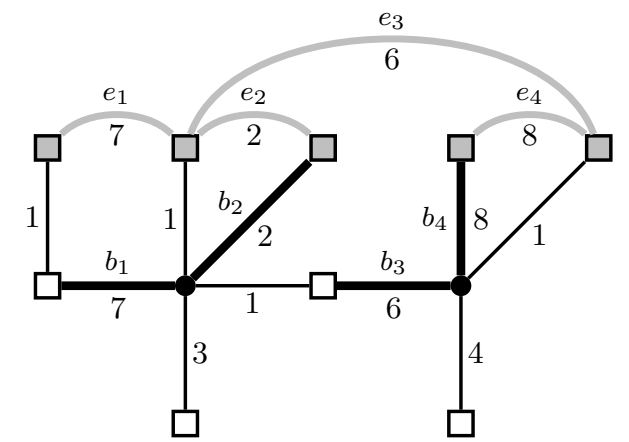

Fig. 2. Steiner tree $S$ is drawn in black. Terminals of $R^{\prime}$ are gray shaded. Bold black edges indicate $\operatorname{Br}_{S}\left(R^{\prime}\right)=\left\{b_{1}, \ldots, b_{4}\right\}$. The corresponding edges $e_{1}, \ldots, e_{4}$ of $Y$ are drawn in gray and labeled with $w\left(e_{i}\right)$. Note that $w\left(e_{i}\right)=c\left(b_{i}\right)$. Observe also that $b_{3}$ is the unique bridge on the cycle contained in $S \cup\left\{e_{3}\right\}$.

A key ingredient in the proof of our lemma is the construction of a proper weighted terminal spanning tree $Y$. Consider a Steiner tree $S$ on terminals $R$. We define a bridge weight function $w: R \times R \rightarrow \mathbb{Q}_{+}$as follows: For any terminal pair $u, v \in R$, the quantity $w(u, v)$ is the maximum cost of any edge in the unique $u-v$ path in $S$. Recall that $\operatorname{Br}_{S}\left(R^{\prime}\right)$ is the set of bridges of $S$ with respect to terminals $R^{\prime}$, and $\mathrm{br}_{S}\left(R^{\prime}\right)$ denotes its cost.

Intuitively, we use the function $w$ to answer the question: If we contract a component containing terminals $R^{\prime}$, what is the cost of edges that we can delete from $S$ ? For example if $R^{\prime}=\{u, v\}$ contains only two terminals, then after contracting $\{u, v\}$, we could delete the most costliest edge on the created cycle in $S$, i.e. $\operatorname{br}_{S}(\{u, v\})=w(u, v)$. The situation becomes slightly more complicated if $\left|R^{\prime}\right|>2$, so we deal with it in the following lemma.

Lemma 10. Let $S$ be any Steiner tree on terminals $R$, and $w: R \times R \rightarrow \mathbb{Q}_{+}$be the associated bridge weight function. For any subset $R^{\prime} \subseteq R$ of terminals, there is a tree $Y \subseteq R^{\prime} \times R^{\prime}$ such that

(a) $Y$ spans $R^{\prime}$.

(b) $w(Y)=\operatorname{br}_{S}\left(R^{\prime}\right)$.

(c) For any $\{u, v\} \in Y$, the $u-v$ path in $S$ contains exactly one edge from $\operatorname{Br}_{S}\left(R^{\prime}\right)$.

Proof. Let $\operatorname{Br}_{S}\left(R^{\prime}\right)=\left\{b_{1}, b_{2}, \ldots, b_{k-1}\right\}$ be the set of bridges. Observe that $S \backslash \operatorname{Br}_{S}\left(R^{\prime}\right)$ is a forest of trees $F_{1}, \ldots, F_{k}$, where each $F_{i}$ contains exactly one terminal $r_{i} \in R^{\prime}$. Each bridge $b_{i}$ connects exactly two trees $F_{i^{\prime}}$ and $F_{i^{\prime \prime}}$. For each $b_{i}$, we add edge $e_{i}=\left\{r_{i^{\prime}}, r_{i^{\prime \prime}}\right\}$ to $Y$. Observe that $Y$ contains $k$ nodes and $k-1$ edges. Assume by contradiction that $Y$ contains a cycle, say $e_{1}, e_{2}, \ldots, e_{g}$. Replace each $e_{i}=\left\{r_{i^{\prime}}, r_{i^{\prime \prime}}\right\}$ with $F_{i^{\prime}} \cup F_{i^{\prime \prime}} \cup\left\{b_{i}\right\}$ : the resulting graph is a cyclic subgraph of $S$, a contradiction. Hence $Y$ is a spanning tree on $R^{\prime}$.

The path $P_{i}$ between $r_{i^{\prime}}$ and $r_{i^{\prime \prime}}$ contains $b_{i}$ and no other bridge. Hence $b_{i}$ is a maximum-cost edge on $P_{i}$, and $w\left(e_{i}\right)=c\left(b_{i}\right)$ (see Figure 2). The claim follows.

The last lemma accounted for the cost reduction, when contracting a single component spanning terminals $R^{\prime}$. Now, we select and contract a component at random

Journal of the ACM, Vol. ?, No. ?, ? 20?. 
from a fractional solution $x$. We will show next, that the expected cost of the edges that we can remove from a tree $T$ is at least $\frac{c(T)}{\sum_{C} x_{C}}$ (interestingly, this only holds, if $T$ is a terminal spanning tree).

Lemma 11. [Bridge Lemma] Let $T$ be a terminal spanning tree and $(x, \mathbf{C})$ be a feasible solution to DCR. Then

$$
c(T) \leq \sum_{C \in \mathbf{C}} x_{C} \cdot \operatorname{br}_{T}(C) .
$$

Proof. For every component $C \in \mathbf{C}$, we construct a spanning tree $Y_{C}$ on $R(C)$ with weight $w\left(Y_{C}\right)=\operatorname{br}_{T}(C)$ according to Lemma 10. Then we direct the edges of $Y_{C}$ towards $\operatorname{sink}(C)$. Let us install cumulatively capacity $x_{C}$ on the (directed) edges of $Y_{C}$, for each $C \in \mathbf{C}$. This way we obtain a directed capacity reservation $y: R \times R \rightarrow \mathbb{Q}_{+}$, with $y(u, v):=\sum_{Y_{C} \ni(u, v)} x_{C}$. The directed tree $Y_{C}$ supports at least the same flow as component $C$ with respect to $R(C)$. It then follows that $y$ supports one unit of flow from each terminal to the root. In other terms, $y$ is a feasible fractional solution to BCR. By Edmonds' Theorem (Theorem 2), BCR is integral when no Steiner node is used. As a consequence there is an (integral) terminal spanning tree $F$ that is not more costly than the fractional solution $y$, i.e., $w(F) \leq \sum_{e \in R \times R} w(e) y(e)$.

Recall that $w(u, v)$, for $u, v \in R$, is the maximum cost of any edge of the unique cycle in $T \cup\{u, v\}$. It follows from the classic cycle rule for minimum spanning tree computation that ${ }^{6} w(F) \geq c(T)$. Altogether

$$
\sum_{C \in \mathbf{C}} x_{C} \operatorname{br}_{T}(C)=\sum_{C \in \mathbf{C}} x_{C} w\left(Y_{C}\right)=\sum_{e \in R \times R} w(e) y(e) \geq w(F) \geq c(T) .
$$

\section{ITERATIVE RANDOMIZED ROUNDING}

In this section we present our approximation algorithm for Steiner tree. To highlight the novel ideas of the approximation technique more than the approximation factor itself, we present a simplified analysis providing a weaker $3 / 2+\varepsilon$ approximation factor (which is already an improvement on the previous best 1.55 approximation). The more complex analysis leading to $\ln (4)+\varepsilon$ is postponed to Section 5 .

The approximation algorithm for Steiner tree is described in Figure 3. In Step (1a) we use the algorithm from Lemma 9. Recall that the cardinality of $\mathbf{C}^{t}$ is upperbounded by a value $M$ which, for any fixed $\varepsilon>0$, is bounded by a polynomial in $n$. Contracting a component $C^{t}$ means collapsing all its terminals into its sink $\operatorname{sink}\left(C^{t}\right)$, which inherits all the edges incident to $C^{t}$ (in case of parallel edges, we only keep the cheapest one). We let $\mathrm{Opt}^{t}$ denote the optimal Steiner tree at the beginning of iteration $t$, and let opt ${ }^{t}$ be its cost. By opt ${ }_{f}^{t}$ we denote the cost of the optimal fractional solution at the beginning of iteration $t$.

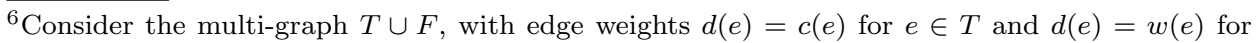
$e \in F$. A minimum spanning tree of this multi-graph can be obtained by removing edges one by one according to the cycle-rule until no cycle is left. Let us apply this algorithm by taking any residual edge $f=u v \in F$, and considering the cycle $C_{f}$ given by $f$ plus the path between $u$ and $v$ in $T$. Each time $f$ can be removed since, by definition of $w(f)=d(f), f$ is the most expensive edge in $C_{f}$. Hence $T$ is a minimum spanning tree. Since $F$ is a spanning tree as well, $c(T)=d(T) \leq d(F)=w(F)$. 
(1) For $t=1,2, \ldots$

(1a) Compute a $\left(1+\frac{\varepsilon}{2}\right)$-approximate solution $\left(x^{t}, \mathbf{C}^{t}\right)$ to DCR (w.r.t. the current instance).

(1b) Sample one component $C^{t}$, where $C^{t}=C$ with probability $x_{C}^{t} / \sum_{C^{\prime} \in \mathbf{C}^{t}} x_{C^{\prime}}^{t}$. Contract $C^{t}$ into its sink.

(1c) If a single terminal remains, return $\bigcup_{i=1}^{t} C^{i}$.

Fig. 3. A $(\ln (4)+\varepsilon)$-approximation algorithm for Steiner tree.

Observe that $\sum_{C \in \mathbf{C}^{t}} x_{C}^{t} \leq M$, and this quantity might vary over the iterations $t$ (in fact, [Chakrabarty et al. 2010a] show that the cardinality of the support of any basic solution is even bounded by $|R|)$. In order to simplify the approximation factor analysis, we consider a dummy algorithm which is the same as the original one, where we replace DCR with the following modified LP. We introduce a dummy component $\bar{C}$ formed by the root only (hence of cost zero), and we add the following equation to our LP:

$$
\sum_{C \in \mathbf{C}^{t}} x_{C}^{t}=M
$$

Observe that the new LP has the same optimal value as before, hence any claim on the integrality gap of the new LP also holds for the original one. Intuitively, the dummy algorithm behaves like the original one except for some idle iterations where the dummy component is sampled. By a simple coupling argument ${ }^{7}$, all the expected properties of the output are the same in the original and dummy algorithm. In particular, any bound on the approximation factor of the dummy algorithm also holds for the original one. Let us remark that, with respect to the running time analysis, one should consider the original algorithm only ${ }^{8}$.

The expected cost of the produced solution is:

$$
\sum_{t \geq 1} E\left[c\left(C^{t}\right)\right] \leq \sum_{t \geq 1} \sum_{C \in \mathbf{C}^{t}} E\left[\frac{x_{C}^{t}}{M} c(C)\right] \leq \frac{1+\frac{\varepsilon}{2}}{M} \sum_{t \geq 1} E\left[\mathrm{opt}_{f}^{t}\right] \leq \frac{1+\frac{\varepsilon}{2}}{M} \sum_{t \geq 1} E\left[\mathrm{opt}^{t}\right]
$$

Thus, in order to obtain a good approximation guarantee, it suffices to provide a good bound on $E\left[\mathrm{opt}^{t}\right]$.

\subsection{A first bound}

The Bridge Lemma shows that given a terminal spanning tree and contracting a random component from any feasible fractional solution, one can remove a $\frac{1}{M}$ fraction (in terms of cost) of the edges and still obtain a terminal spanning tree. In other words, the cost of the minimum terminal spanning tree decreases by a factor $\left(1-\frac{1}{M}\right)$ per iteration in expectation. This implies an upper bound on $\operatorname{opt}_{f}^{t}$ via Lemma 5 (while later bounds will hold for opt ${ }^{t}$ only). The bound on $\mathrm{opt}_{f}^{t}$ implies

\footnotetext{
${ }^{7}$ The coupling works as follows: for any input sequence $\mathcal{I}$ of random bits for the original algorithm, consider the subsequence $\mathcal{I}^{\prime}$ obtained from $\mathcal{I}$ by discarding random bits used in idle iterations. Observe that $\mathcal{I}^{\prime}$ is a sequence of random bits. We run the dummy algorithm using $\mathcal{I}^{\prime}$ as random bit sequence. This way, the two algorithms output exactly the same solution deterministically. ${ }^{8}$ It is possible to show that the dummy algorithm runs in expected polynomial time, but this is not crucial since the original algorithm needs at most $|R|$ iterations.

Journal of the ACM, Vol. ?, No. ?, ? 20?.
} 
the first non-trivial bounds on the approximation guarantee of our algorithm (due to the fact that $\mathrm{opt}_{f}^{t} \leq \mathrm{opt}^{t}$ ) and on the integrality gap of our LP.

LEMMA 12. $E\left[\mathrm{opt}_{f}^{t}\right] \leq\left(1-\frac{1}{M}\right)^{t-1} \cdot 2 \mathrm{opt}_{f}$.

Proof. Let $T^{t}$ be the minimum-cost terminal spanning tree at the beginning of iteration $t$. By Lemma $5, c\left(T^{1}\right) \leq 2 \operatorname{opt}_{f}$. For any iteration $t>1$, the reduction in the cost of $T^{t}$ w.r.t. $T^{t-1}$ is at least $\mathrm{br}_{T^{t-1}}\left(C^{t}\right)$. Therefore, conditioning over $T^{t-1}$,

$$
\begin{aligned}
& E\left[c\left(T^{t}\right)\right] \leq c\left(T^{t-1}\right)-E\left[b_{T^{t-1}}\left(C^{t-1}\right)\right] \\
& =\quad c\left(T^{t-1}\right)-\frac{1}{M} \sum_{C \in \mathbf{C}^{t-1}} x_{C}^{t-1} \cdot \mathrm{br}_{T^{t-1}}(C) \\
& \stackrel{\text { Bridge Lem } 11}{\leq}\left(1-\frac{1}{M}\right) \cdot c\left(T^{t-1}\right) .
\end{aligned}
$$

By induction on the conditioned expectations,

$$
E\left[\mathrm{opt}_{f}^{t}\right] \leq E\left[c\left(T^{t}\right)\right] \leq\left(1-\frac{1}{M}\right)^{t-1} \cdot 2 \mathrm{opt}_{f} .
$$

Observe that the bound from Lemma 12 improves over the trivial bound opt $_{f}^{t} \leq$ $\mathrm{opt}_{f}$ only for $t>M \cdot \ln (2)$. Nevertheless it suffices to prove the following result.

THEOREM 13. For any fixed $\varepsilon>0$, there is a randomized polynomial-time algorithm which computes a solution to the Steiner tree problem of expected cost at most $(1+\ln (2)+\varepsilon) \cdot$ opt $_{f}$.

Proof. Assume without loss of generality that $M \cdot \ln (2)$ is integral. Combining (1) with Lemma 12, the expected approximation factor is

$$
\begin{aligned}
E\left[\frac{\sum_{t \geq 1} c\left(C^{t}\right)}{\mathrm{opt}_{f}}\right] & \leq \frac{1+\varepsilon / 2}{M} \sum_{t \geq 1} E\left[\frac{\mathrm{opt}_{f}^{t}}{\mathrm{opt}_{f}}\right] \\
& \leq \frac{1+\varepsilon / 2}{M} \sum_{t \geq 1} \min \left\{1,2\left(1-\frac{1}{M}\right)^{t-1}\right\} \\
& \leq \frac{1+\varepsilon / 2}{M}\left(M \cdot \ln (2)+\sum_{t \geq M \cdot \ln (2)+1} 2\left(1-\frac{1}{M}\right)^{t-1}\right) \\
& \leq\left(1+\frac{\varepsilon}{2}\right)\left(\ln (2)+2\left(1-\frac{1}{M}\right)^{M \cdot \ln (2)}\right) \\
& \leq\left(1+\frac{\varepsilon}{2}\right)\left(\ln (2)+2 e^{-\ln (2)}\right) \leq 1+\ln (2)+\varepsilon .
\end{aligned}
$$

Above we used the equation $\sum_{t \geq t_{0}} x^{t}=\frac{x^{t_{0}}}{1-x}$ for $0<x<1$ and the inequality $(1-1 / x)^{x} \leq 1 / e$ for $x \geq 1$.

Observe that Theorem 13 implies that the integrality gap of DCR is at most $1+$ $\ln (2)<1.694$. In Section 7 we will refine this bound on the gap to $1+\ln (3) / 2<1.55$. 


\subsection{A second bound}

In order to further improve the approximation guarantee we show that, in each iteration, the cost opt ${ }^{t}$ of the optimal (integral) Steiner tree of the current instance decreases by a factor $\left(1-\frac{1}{2 M}\right)$ in expectation. We remark that it is not known whether this bound holds also for opt $f_{f}^{t}$. Also in this case the proof relies crucially on the Bridge Lemma.

Lemma 14. Let $S$ be any Steiner tree and $(x, \mathbf{C})$ be a feasible solution to DCR. Sample a component $C \in \mathbf{C}$ such that $C=C^{\prime}$ with probability $x_{C^{\prime}} / M$. Then there is a subgraph $S^{\prime} \subseteq S$ such that $S^{\prime} \cup C$ spans $R$ and

$$
E\left[c\left(S^{\prime}\right)\right] \leq\left(1-\frac{1}{2 M}\right) \cdot c(S)
$$

Proof. It suffices to prove that $E\left[\operatorname{br}_{S}(C)\right] \geq \frac{1}{2 M} c(S)$. Turn $S$ into a rooted binary tree with the usual procedure. Then, for any Steiner node in $S$, choose the cheapest edge going to one of its children. The set $H \subseteq S$ of such selected edges has cost $c(H) \leq \frac{1}{2} c(S)$. Furthermore any Steiner node is connected to one terminal using edges of $H$. Consider the terminal spanning tree $T$ that emerges from $S$ by contracting $H$. By the Bridge Lemma 11,

$$
E\left[\operatorname{br}_{T}(C)\right]=\frac{1}{M} \sum_{C^{\prime} \in \mathbf{C}} x_{C^{\prime}} \cdot \operatorname{br}_{T}\left(C^{\prime}\right) \geq \frac{1}{M} c(T) .
$$

Note that for any set of edges $B \subseteq T$ and any component $C$ the following holds: If $(T \backslash B) \cup C$ connects $R$, then also $(S \backslash B) \cup C$ connects $^{9} R$. This implies that $\operatorname{br}_{S}(C) \geq \operatorname{br}_{T}(C)$. Altogether:

$$
E\left[\operatorname{br}_{S}(C)\right] \geq E\left[\operatorname{br}_{T}(C)\right] \geq \frac{1}{M} c(T)=\frac{1}{M}(c(S)-c(H)) \geq \frac{1}{2 M} c(S) .
$$

Iterating Lemma 14 yields the following corollary.

COROLlary 15. For every $t \geq 1$,

$$
E\left[\mathrm{opt}^{t}\right] \leq\left(1-\frac{1}{2 M}\right)^{t-1} \cdot \text { opt }
$$

We now have all the ingredients to show a $(3 / 2+\varepsilon)$-approximation factor.

THEOREM 16. For any $\varepsilon>0$, there is a polynomial-time randomized approximation algorithm for Steiner tree with expected approximation ratio $3 / 2+\varepsilon$.

Proof. Assume without loss of generality that $M \cdot \ln (4)$ is integral. Combining

\footnotetext{
${ }^{9}$ Using that for any edge $e \in T$ between terminals $u, v \in R$, there exists a path from $u$ to $v$ in $S$, which uses only edge $e$ plus some edges in $H$.

Journal of the ACM, Vol. ?, No. ?, ? 20?.
} 
(1) with Lemma 12 and Corollary 15, the expected approximation factor is:

$$
\begin{aligned}
E\left[\frac{\sum_{t \geq 1} c\left(C^{t}\right)}{\mathrm{opt}}\right] & \leq \frac{1+\varepsilon / 2}{M} \sum_{t \geq 1} E\left[\frac{\mathrm{opt}^{t}}{\mathrm{opt}}\right] \\
& \leq \frac{1+\varepsilon / 2}{M} \sum_{t \geq 1} \min \left\{2\left(1-\frac{1}{M}\right)^{t-1},\left(1-\frac{1}{2 M}\right)^{t-1}\right\} \\
& \leq \frac{1+\varepsilon / 2}{M}\left(\sum_{t=1}^{M \cdot \ln (4)}\left(1-\frac{1}{2 M}\right)^{t-1}+\sum_{t \geq M \cdot \ln (4)+1} 2\left(1-\frac{1}{M}\right)^{t-1}\right) \\
& =\left(1+\frac{\varepsilon}{2}\right) \cdot\left(2-2 \cdot\left(1-\frac{1}{2 M}\right)^{M \cdot \ln (4)}+2\left(1-\frac{1}{M}\right)^{M \cdot \ln (4)}\right) \\
& \leq\left(1+\frac{\varepsilon}{2}\right) \cdot\left(2-2 \cdot e^{-\ln (4) / 2}+2 e^{-\ln (4)}\right) \leq 3 / 2+\varepsilon .
\end{aligned}
$$

Above we exploited the equation $\sum_{t=1}^{t_{0}} x^{t-1}=\frac{1-x^{t_{0}}}{1-x}$ for $0<x<1$. We also used the fact that $\left(1-\frac{1}{y}\right)^{\ln (4) y}-\left(1-\frac{1}{2 y}\right)^{\ln (4) y}$ is an increasing function of $y>1$, and that $\lim _{y \rightarrow \infty}\left(1-\frac{1}{y}\right)^{y}=\frac{1}{e}$.

\section{A REFINED ANALYSIS}

In this section we present a refined $(\ln (4)+\varepsilon)$ approximation bound for our Steiner tree algorithm.

We first give a high-level description of our analysis. Let $S^{*}:=$ Opt be the optimal Steiner tree for the original instance (in particular, $c\left(S^{*}\right)=\mathrm{opt}$ ). Each time we sample a component $C^{t}$, we will delete a proper subset of edges from $S^{*}$. Consider the sequence $S^{*}=S^{1} \supseteq S^{2} \supseteq \ldots$ of subgraphs of $S^{*}$ which are obtained this way. We will guarantee that at any iteration $t$, the edge set $S^{t}$ plus the previously sampled components yields a subgraph that connects all terminals. Furthermore, we will prove that a fixed edge $e \in S^{*}$ is deleted after an expected number of at most $\ln (4) \cdot M$ iterations. This immediately implies the approximation factor of $\ln (4)+\varepsilon$.

In order to track which edges can be safely deleted from $S^{*}$, we will construct an artificial terminal spanning tree $W$ (the witness tree) and assign a random subset $W(e)$ of edges of $W$ to each edge $e \in S^{*}$ (the witnesses of $e$ ). At each iteration, when component $C^{t}$ is sampled, we mark a proper random subset $\overline{\mathrm{Br}}_{W}\left(C^{t}\right)$ of edges of $W$; we will later define our choice of subset $\overline{\mathrm{Br}}_{W}\left(C^{t}\right)$. As soon as all the edges of $W(e)$ are marked, edge $e$ is deleted from $S^{*}$. Summarizing, we consider the following random process:

For $t=1,2, \ldots$, sample one component $C^{t}$ from $\left(x^{t}, \mathbf{C}^{t}\right)$ and mark the edges in $\overline{\mathrm{Br}}_{W}\left(C^{t}\right)$. Delete an edge $e$ from $S^{*}$ as soon as all edges in $W(e)$ are marked.

The subgraph $S^{t}$ is formed by the edges of $S^{*}$ which are not yet deleted at the beginning of iteration $t$.

Our choice of $\overline{\mathrm{Br}}_{W}\left(C^{t}\right)$ will guarantee that, deterministically, the unmarked edges $W^{\prime}$ plus the sampled components connect all the terminals. Our choice of $W(e)$ 

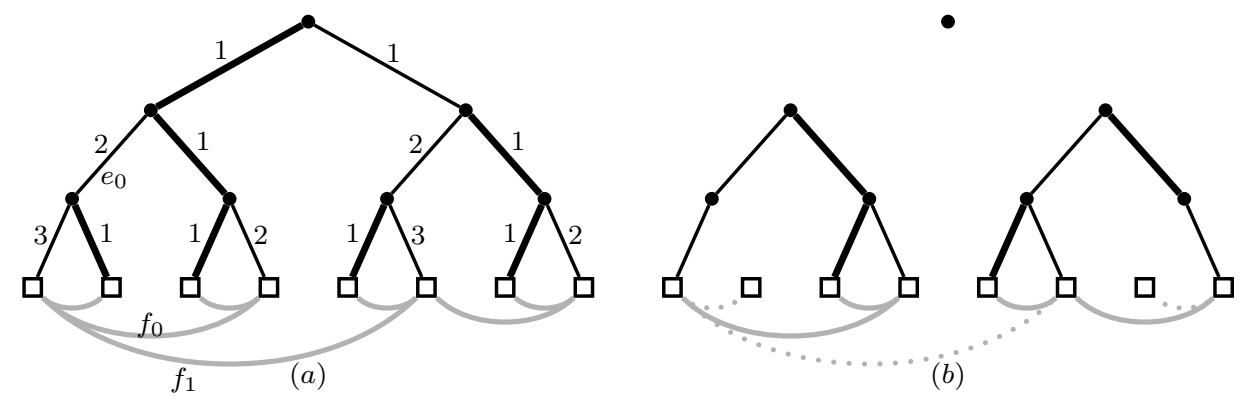

Fig. 4. (a) Optimal Steiner tree $S^{*}$ in black, where bold edges indicate the chosen edges $\tilde{B}$, and the associated terminal spanning tree $W$ in gray. Edges $e$ in $S^{*}$ are labeled with $|W(e)|$. For example $W\left(e_{0}\right)=\left\{f_{0}, f_{1}\right\}$. (b) Marked edges in $W$ at a given iteration $t$ are drawn dotted; the non-deleted edges in $S^{*}$ (i.e., edges of $S^{t}$ ) are drawn in black. Non-marked edges of $W$ and non-deleted edges of $S^{*}$ support the same connectivity on $R$.

will ensure that, deterministically, if $W^{\prime}$ plus the sampled components connect all the terminals, then the sampled components plus the undeleted edges $S^{t}=\{e \in$ $\left.S^{*} \mid W(e) \cap W^{\prime} \neq \emptyset\right\}$ do the same. Hence the $S^{t}$ 's have the claimed connectivity properties. The analysis then reduces to show that all the edges in $W(e)$ are marked within a small enough number of iterations (in expectation).

We next define $W, W(\cdot)$, and $\overline{\mathrm{Br}}_{W}(\cdot)$. Turn $S^{*}$ into a rooted binary tree with the usual procedure. Recall that the height of the binary tree is at most $|R|-1$. For each Steiner node, choose uniformly at random one of the two edges to its children. Let $\tilde{B}$ denote the chosen edges. Clearly $\operatorname{Pr}[e \in \tilde{B}]=\frac{1}{2}$ for any $e \in S^{*}$. Let $P_{u v} \subseteq S^{*}$ be the unique $u-v$ path in $S^{*}$. The witness tree is

$$
W:=\left\{\{u, v\} \in\left(\begin{array}{c}
R \\
2
\end{array}\right)|| P_{u v} \cap \tilde{B} \mid=1\right\} .
$$

As in the arguments of Lemma $10, W$ is a terminal spanning tree. For each edge $e \in S^{*}$, define

$$
W(e):=\left\{\{u, v\} \in W \mid e \in P_{u v}\right\} .
$$

See Figure 4(a) for an illustration. As we will see, $W(e)$ is small in expectation. It remains to define $\overline{\mathrm{Br}}_{W}(\cdot)$. For a given component $C \in \mathbf{C}$, let the set of candidate bridges $\mathcal{B}_{W}(C)$ be

$$
\mathcal{B}_{W}(C):=\{B \subseteq W|| B|=| R(C) \mid-1,(W \backslash B) \cup C \text { connects } V(W)\} .
$$

Intuitively, $\mathcal{B}_{W}(C)$ is the family of bridge sets of $W$ with respect to $C$ that one obtains for varying cost functions. The set $\overline{\mathrm{Br}}_{W}\left(C^{t}\right)$ is chosen randomly in $\mathcal{B}_{W}(C)$, according to a proper probability distribution $w_{C}: \mathcal{B}_{W}(C) \rightarrow[0,1]$, which will be described in the following. Observe that $\operatorname{Br}_{W}(C) \in \mathcal{B}_{W}(C)$. The intuitive reason for using a random element of $\mathcal{B}_{W}(C)$ rather than $\operatorname{Br}_{W}(C)$ is that we wish to mark the edges of $W$ in a more uniform way. This, in combination with the small size of $W(e)$, guarantees that edges are deleted quickly enough.

The next lemma shows that the undeleted edges plus the sampled components connect the terminals.

Journal of the ACM, Vol. ?, No. ?, ? 20?. 
Lemma 17. The graph $S^{t} \cup \bigcup_{t^{\prime}=1}^{t-1} C^{t^{\prime}}$ spans $R$.

Proof. Let $W^{\prime} \subseteq W$ be the set of edges which are not yet marked at the beginning of iteration $t$ (see also Figure $4(\mathrm{~b})$ ). By the definition of $\mathcal{B}_{W}(C)$ and since $\overline{\mathrm{Br}}_{W}(C) \in \mathcal{B}_{W}(C), W^{\prime} \cup \bigcup_{t^{\prime}=1}^{t-1} C^{t^{\prime}}$ spans $R$. Consider any edge $\{u, v\} \in W^{\prime}$. Then $\{u, v\} \in W(e)$ for all $e \in P_{u v}$. Hence no edge on $P_{u v}$ is deleted. Therefore $u$ and $v$ are also connected in $S^{t}$. The claim follows.

Note that $1 \leq|W(e)| \leq|R|-1$. Observe also that $|W(e)|=1$ if $e \in \tilde{B}$. Indeed, the expected cardinality of $W(e)$ is small also for the remaining edges.

Lemma 18. For any edge $e \in S^{*}$ at level $k_{e} \leq|R|-1$ (edges incident to the root are at level one), one has

$$
\operatorname{Pr}[|W(e)|=q]= \begin{cases}1 / 2^{q} & \text { if } 1 \leq q<k_{e} \\ 2 / 2^{q} & \text { if } q=k_{e} \\ 0 & \text { otherwise }\end{cases}
$$

Proof. Consider the path $v_{0}, v_{1}, \ldots, v_{k_{e}}$ from $e$ towards the root. In particular, $e=\left\{v_{0}, v_{1}\right\}$. If $\left(v_{q-1}, v_{q}\right)$ is the first edge from $\tilde{B}$ on this path, then $|W(e)|=q$. This is because, for each node $v_{j}, j \in\left\{1, \ldots, k_{e}\right\}$, there is one distinct path $P_{u v}$, with $\{u, v\} \in W$ and having $v_{j}$ as its top node, that contains $e$ (see also Figure $4(\mathrm{a}))$. This event happens with probability $1 / 2^{q}$. If there is no edge from $\tilde{B}$ on the path $v_{0}, v_{1}, \ldots, v_{k_{e}},|W(e)|=k_{e}$ by a similar argument. The latter event happens with probability $1 / 2^{k_{e}}$. The claim follows.

Next lemma proves the existence of random variables $\overline{\mathrm{Br}}_{W}(\cdot)$ such that each edge of $W$ is marked at each iteration with probability at least $1 / M$. Its proof is based on a combination of Farkas' Lemma with our Bridge Lemma.

LEMma 19. There is a choice of the random variables $\overline{\mathrm{Br}}_{W}(\cdot)$ such that each edge $e \in W$ is marked with probability at least $1 / M$ at each iteration.

Proof. Consider any given iteration. Let $(x, \mathbf{C})$ be the corresponding solution to DCR, and $C^{*}$ be the sampled component in that iteration. In particular, $C^{*}=C$ with probability $x_{C} / M=x_{C} / \sum_{C^{\prime} \in \mathbf{C}} x_{C^{\prime}}$. In this iteration we mark the edges $\overline{\mathrm{Br}}_{W}\left(C^{*}\right)$, where $\operatorname{Pr}\left[\overline{\mathrm{Br}}_{W}\left(C^{*}\right)=B\right]=w_{C^{*}}(B)$ for any $B \in \mathcal{B}_{W}\left(C^{*}\right)$. We will show that there is a choice of the $w_{C}$ 's, $C \in \mathbf{C}$, such that

$$
\sum_{(C, B): B \in \mathcal{B}_{W}(C), e \in B} x_{C} \cdot w_{C}(B) \geq 1, \quad \forall e \in W .
$$

This implies the claim since

$$
\operatorname{Pr}\left[e \in \overline{\operatorname{Br}}_{W}\left(C^{*}\right)\right]=\sum_{(C, B): B \in \mathcal{B}_{W}(C), e \in B} \frac{x_{C}}{M} \cdot w_{C}(B) \geq \frac{1}{M} .
$$

Suppose by contradiction that such probability distributions $w_{C}$ do not exist. 
Then the following linear system has no solution ${ }^{10}$ :

$$
\begin{aligned}
\sum_{B \in \mathcal{B}_{W}(C)} w_{C}(B) & \leq 1, \quad \forall C \in \mathbf{C} ; \\
\sum_{(C, B): B \in \mathcal{B}_{W}(C), e \in B} x_{C} \cdot w_{C}(B) & \geq 1, \quad \forall e \in W ; \\
w_{C}(B) & \geq 0, \quad \forall C \in \mathbf{C}, \forall B \in \mathcal{B}_{W}(C) .
\end{aligned}
$$

Farkas' Lemma ${ }^{11}$ yields that there is a vector $(y, c) \geq 0$ with

$$
\begin{aligned}
& \text { (a) } y_{C} \geq \sum_{e \in B} c_{e} x_{C}, \quad \forall C \in \mathbf{C}, \forall B \in \mathcal{B}_{W}(C) \text {; } \\
& \text { (b) } \sum_{C \in \mathbf{C}} y_{C}<\sum_{e \in W} c_{e} .
\end{aligned}
$$

Let us interpret $c$ as an edge cost function. In particular, $c(W):=\sum_{e \in W} c_{e}$ and $\operatorname{br}_{W}(C)$ is the cost of the bridges of $W$ with respect to component $C$ and this cost function. One has

$$
y_{C} \stackrel{(a)}{\geq} x_{C} \cdot \max \left\{c(B) \mid B \in \mathcal{B}_{W}(C)\right\}=x_{C} \cdot \operatorname{br}_{W}(C) .
$$

Then

$$
\sum_{C \in \mathbf{C}} x_{C} \cdot \operatorname{br}_{W}(C) \leq \sum_{C \in \mathbf{C}} y_{C} \stackrel{(b)}{<} \sum_{e \in W} c_{e}=c(W),
$$

which contradicts the Bridge Lemma 11.

We next show that, for small $|W(e)|$, all the edges of $W(e)$ are marked (and hence $e$ is deleted) within a small number of iterations. A handwaving argument works as follows. Let $|W(e)|=q$. Similarly to the Coupon Collector problem (see e.g. [Mitzenmacher and Upfal 2005]), it takes in expectation $\frac{M}{q}$ iterations until the first edge is marked, then $\frac{M}{q-1}$ iterations to hit the second one and so forth. Finally all edges are marked after an expected number of $M \cdot\left(\frac{1}{q}+\frac{1}{q-1}+\ldots+1\right)=H_{q} \cdot M$ iterations. (Here $H_{q}:=\sum_{i=1}^{q} \frac{1}{i}$ denotes the $q$-th harmonic number). However, this argument does not reflect the fact that a set $\overline{\mathrm{Br}}_{W}\left(C^{t}\right)$ might contain several edges from $W(e)$. A more careful argument incorporates this complication.

For $\tilde{W} \subseteq W$, let $X(\tilde{W})$ denote the first iteration when all the edges in $\tilde{W}$ are marked. Observe that $S^{t}=\left\{e \in S^{*} \mid X(W(e)) \geq t\right\}$.

Lemma 20. Let $\tilde{W} \subseteq W$. Then the expected number of iterations until all edges in $\tilde{W}$ are marked satisfies

$$
E[X(\tilde{W})] \leq H_{|\tilde{W}|} \cdot M
$$

Proof. Let $q=|\tilde{W}|$. By $m_{q}$ we denote the best possible upper bound on the expected number of iterations until all edges of $\tilde{W}$ are marked (over all feasible probability distributions). We will prove that $m_{q} \leq H_{q} \cdot M$ by induction on $q$.

\footnotetext{
${ }^{10}$ We can replace the "=" constraint with " $\leq$ " without affecting feasibility since all coefficients of $w_{C}(B)$ are non-negative.

${ }^{11} \exists x \geq \mathbf{0}: A x \leq b \dot{\vee} \exists z \geq \mathbf{0}: z^{T} A \geq 0, z^{T} b<0$.

Journal of the ACM, Vol. ?, No. ?, ? 20?
} 
For $q=1$, the only edge in $\tilde{W}$ is marked with probability at least $\frac{1}{M}$ at each iteration, hence $m_{1} \leq M$. Next, let $q>1$ and consider the first iteration. Suppose that $\lambda_{i}$ is the probability that at least $i$ many edges are marked in this iteration. Since the expected number of marked edges must be at least $q \cdot \frac{1}{M}$ in the first iteration, this distribution has to satisfy $\sum_{i=1}^{q} \lambda_{i} \geq \frac{q}{M}$. Note that $\lambda_{0}=1$ and $\lambda_{q+1}=0$. For notational convenience, let $m_{0}:=0$.

If we condition on the event that $i \in\{0, \ldots, q\}$ edges are marked in the first iteration, we need in expectation at most $m_{q-i}$ more iterations until the remaining $q-i$ edges are marked. Hence we obtain the following bound:

$$
\begin{aligned}
m_{q} & \leq 1+\sum_{i=0}^{q} \operatorname{Pr}\left[\begin{array}{c}
\text { exactly } i \text { edges marked } \\
\text { at the first iteration }
\end{array}\right] \cdot m_{q-i} \\
\begin{array}{c}
\text { inductive } \\
\text { hypothesis }
\end{array} & 1+M \cdot \sum_{i=1}^{q}\left(\lambda_{i}-\lambda_{i+1}\right) H_{q-i}+\left(1-\lambda_{1}\right) m_{q} \\
& =1+M \cdot \sum_{i=1}^{q} \lambda_{i} \cdot \underbrace{\left(H_{q-i}-H_{q-i+1}\right)}_{\leq-1 / q}+\lambda_{1} H_{q} M+\left(1-\lambda_{1}\right) m_{q} \\
& \leq 1-\frac{1}{q} M \cdot \underbrace{\sum_{i=1}^{q} \lambda_{i}}_{\geq q / M}+\lambda_{1} H_{q} M+\left(1-\lambda_{1}\right) m_{q} \\
& \leq \quad \lambda_{1} H_{q} M+\left(1-\lambda_{1}\right) m_{q} .
\end{aligned}
$$

From $\lambda_{1}>0$ we obtain $m_{q} \leq H_{q} \cdot M$. The claim follows.

Now we have all the ingredients to prove the expected $(\ln (4)+\varepsilon)$ approximation factor.

THEOREM 21. For any constant $\varepsilon>0$, there is a polynomial-time randomized approximation algorithm for the Steiner tree problem with expected approximation ratio $\ln (4)+\varepsilon$.

Proof. For an edge $e \in S^{*}$, we define $D(e)=\max \left\{t \mid e \in S^{t}\right\}$ as the iteration Journal of the ACM, Vol. ?, No. ?, ? 20?. 
in which $e$ is deleted. One has ${ }^{12}$

$$
\begin{aligned}
E[D(e)] & =\sum_{q=1}^{k_{e}} \operatorname{Pr}[|W(e)|=q] \cdot E[D(e)|| W(e) \mid=q] \\
\stackrel{\mathrm{Lem}}{\leq} 20 & \sum_{q=1}^{k_{e}} \operatorname{Pr}[|W(e)|=q] \cdot H_{q} \cdot M \\
\stackrel{\mathrm{Lem}}{=} 18 & \sum_{q=1}^{k_{e}-1}\left(\frac{1}{2}\right)^{q} \cdot H_{q} \cdot M+\frac{2}{2^{k_{e}}} \cdot H_{k_{e}} \cdot M \\
& \leq \sum_{q \geq 1}\left(\frac{1}{2}\right)^{q} \cdot H_{q} \cdot M \\
& =M \cdot \sum_{q \geq 1} \frac{1}{q} \sum_{i \geq 0}\left(\frac{1}{2}\right)^{q+i} \\
& =M \cdot \sum_{q \geq 1} \frac{1}{q}\left(\frac{1}{2}\right)^{q-1}=\ln (4) \cdot M .
\end{aligned}
$$

The expected cost of the approximate solution satisfies

$$
\begin{aligned}
E\left[\sum_{t \geq 1} c\left(C^{t}\right)\right] & \leq \sum_{t \geq 1} \frac{1+\varepsilon / 2}{M} E\left[\mathrm{opt}_{f}^{t}\right] \\
& \leq \frac{1+\varepsilon / 2}{M} \sum_{t \geq 1} E\left[c\left(S^{t}\right)\right] \\
& =\frac{1+\varepsilon / 2}{M} \sum_{e \in S^{*}} E[D(e)] \cdot c(e) \leq(\ln (4)+\varepsilon) \cdot \mathrm{opt} .
\end{aligned}
$$

The claim follows.

\subsection{A $\left(\frac{73}{60}+\varepsilon\right)$-Approximation for Quasi-Bipartite Graphs}

In this section we consider the special case of quasi-bipartite graphs. Recall that we call a graph $G=(V, E)$ quasi-bipartite if no pair of non-terminal nodes $u, v \in V \backslash R$ is connected by an edge. In other words, the edge sets is a union of stars (with a Steiner node as center and terminals as leaves) which only share common terminals (plus edges running directly between terminals). We show that our algorithm has an approximation ratio of at most $\frac{73}{60}+\varepsilon<1.217$ (for $\varepsilon$ small enough). This improves over the previously best known factor of 1.28 in [Robins and Zelikovsky 2005]. Note that Gröpl et al. [2002] show the bound of $\frac{73}{60}$ for the more restricted case of uniform quasi-bipartite graphs, where all edges incident to a non-terminal node have the same cost. For this class the integrality gap of the hypergraphic LP relaxation by Chakrabarty et al. [2010a] can also be bounded by $\frac{73}{60}$.

${ }^{12} L i_{n}(z):=\sum_{q \geq 1} \frac{z^{q}}{q^{n}}$ is known as the polylogarithm or Jonquière's function. It is well-known that $L i_{1}(z)=\ln \left(\frac{1}{1-z}\right)$, from which we obtain in the final equation: $\sum_{q \geq 1} \frac{1}{q}\left(\frac{1}{2}\right)^{q-1}=2 \sum_{q \geq 1} \frac{1}{q}\left(\frac{1}{2}\right)^{q}=$ $2 \ln \left(\frac{1}{1-1 / 2}\right)=\ln (4)$.

Journal of the ACM, Vol. ?, No. ?, ? 20?. 
Let $S^{*}$ be an optimal Steiner tree, and let $Z_{1}, \ldots, Z_{\ell}$ be its (undirected) components. Since the input graph (and hence $S^{*}$ ) is quasi-bipartite, each $Z_{i}$ (unless it consists of a single edge) must be a star with a single Steiner node as center and terminals as leaves. In fact otherwise there would be two adjacent internal (and hence non-terminal) nodes in $Z_{i}$. We can improve the approximation guarantee by choosing the witness tree $W$ in a more economical way, exploiting the structure of $S^{*}$. For each $i=1, \ldots, \ell$, we add to the chosen edges $\tilde{B}$ all the edges of $Z_{i}$ but one edge chosen uniformly at random. Again for $u, v \in R$, let $P_{u v}$ be the unique $u-v$ path in $S^{*}$. We let

$$
W:=\left\{\{u, v\} \in\left(\begin{array}{c}
R \\
2
\end{array}\right)|| P_{u v} \cap \tilde{B} \mid=1\right\} .
$$

Observe that $W$ will in fact be a terminal spanning tree. The analysis is now much simpler.

ThEOREM 22. For any constant $\varepsilon>0$, there is a polynomial-time randomized approximation algorithm for the Steiner tree problem on quasi-bipartite graphs with expected approximation ratio $\frac{73}{60}+\varepsilon$.

Proof. We still consider the algorithm in Figure 3. For an edge $e \in S^{*}$, we define $D(e)=\max \left\{t \mid e \in S^{t}\right\}$ as the iteration in which $e$ is deleted. Let $k$ be the number of terminals in the star $Z_{i}$ that contains $e$. With probability $\frac{1}{k}$ one has $|W(e)|=k-1$, and otherwise $|W(e)|=1$. Hence, by Lemma 20,

$E[D(e)] \leq \frac{1}{k} \cdot H_{k-1} \cdot M+\left(1-\frac{1}{k}\right) \cdot H_{1} \cdot M=\left(\frac{1}{k} \cdot H_{k-1}+\frac{k-1}{k}\right) \cdot M \leq \frac{73}{60} \cdot M$.

In the last inequality we used the fact ${ }^{13}$ that $\frac{1}{k} \cdot H_{k-1}+\frac{k-1}{k}$ is maximized for $k=5$. The claim follows along the same line as in Theorem 21 .

\section{DERANDOMIZATION}

In this section, we show how to derandomize the result from Section 5 using the method of limited independence (see, e.g., [Alon and Spencer 2008]). This way, we prove Theorem 3.

We start (Section 6.1) by presenting an alternative, phase-based algorithm, which updates the LP only a constant number of times (the phases). Then we show (Section 6.2) how to sample components in each phase with a logarithmic number of random bits.

\subsection{A Phase-Based Randomized Algorithm}

Consider the algorithm from Figure 5. The basic idea behind the algorithm is grouping iterations into phases. In each phase, we keep the LP unchanged. The details on how to sample components in each phase are given later.

We may assume that the computed DCR solution $\left(x^{s}, \mathbf{C}^{s}\right)$ is well-rounded, i.e.,

- $\left|\mathbf{C}^{s}\right|=m$ for a prime number $m$,

- $x_{C}^{s}=\frac{1}{N}$ for all $C \in \mathbf{C}^{s}$ and $N \geq 1$ is bounded by a polynomial in $n$.

${ }^{13}$ To see that, let $f(k):=\frac{1}{k} \cdot H_{k-1}+\frac{k-1}{k}$. The difference $f(k+1)-f(k)=\frac{2-H_{k-1}}{k(k+1)}$ is positive for $k \leq 4$ and negative for $k \geq 5$. 
(1) For phase $s=1,2, \ldots, 1 / \varepsilon^{2}$

(1a) Compute a $(1+\varepsilon)$-approximate well-rounded solution $\left(x^{s}, \mathbf{C}^{s}\right)$ to DCR (w.r.t. the current instance).

(1b) Sample $\phi^{s}$ components $C^{s, 1}, \ldots, C^{s, \phi^{s}}$ from $\mathbf{C}^{s}$ according to $x^{s}$, and contract them.

(2) Compute a minimum-cost terminal spanning tree $T$ in the remaining instance.

(3) Output $T \cup \bigcup_{s=1}^{1 / \varepsilon^{2}} \bigcup_{i=1}^{\phi^{s}} C^{s, i}$.

Fig. 5. Phase-based sampling algorithm

This can be achieved as follows: One computes a $\left(1+\frac{\varepsilon}{2}\right)$-approximate solution $(x, \mathbf{C})$. Say $h=|\mathbf{C}|$. Then we round up all entries in $x$ to the nearest multiple of $\frac{1}{N}$ for $N:=8 h / \varepsilon$ and term the obtained solution $x^{\prime}$. Using the generous estimate $c(C) \leq 2 \operatorname{opt}_{f}$ (following from Lemma 5) we obtain, for $\varepsilon \leq 1$,

$$
\sum_{C \in \mathbf{C}} x_{C}^{\prime} \cdot c(C) \leq\left(1+\frac{\varepsilon}{2}\right) \cdot\left(\mathrm{opt}_{f}+\sum_{C \in \mathbf{C}} \frac{\varepsilon}{8 h} c(C)\right) \leq(1+\varepsilon) \operatorname{opt}_{f} .
$$

Next, replace a component $C$ by $x_{C}^{\prime} \cdot N$ many copies. Let $m^{\prime}$ be the number of obtained components (counted with multiplicities). Then we can compute a prime number $m \in\left[m^{\prime}, 2 m^{\prime}\right]$ (see e.g. [Niven et al. 1991]) and add $m-m^{\prime}$ dummy components $C$ containing only the root, each one with $x_{C}^{\prime}:=\frac{1}{N}$. This yields a feasible well-rounded solution as desired. We furthermore assume ${ }^{14}$ that $m \geq N / \varepsilon^{2}$ and $1 / \varepsilon$ is integer.

For $\tilde{W} \subseteq W$, let $\bar{X}(\tilde{W})$ denote the first phase when all edges in $\tilde{W}$ are marked. Analogously, $\bar{D}(e)$ is the phase when all the edges in $W(e)$ are marked. For notational convenience, we interpret Step (2) as a final phase when all the edges of $W$ are marked (so that $\bar{X}(\tilde{W})$ and $\bar{D}(e)$ are well defined). The next lemma is a simple adaptation of Lemma 20 .

Lemma 23. Let $\tilde{W} \subseteq W$. Suppose each edge is marked at each phase with probability at least $p \in(0,1]$. Then the expected number of phases until all edges in $\tilde{W}$ are marked satisfies

$$
E[\bar{X}(\tilde{W})] \leq H_{|\tilde{W}|} \cdot \frac{1}{p}
$$

Proof. By a simple coupling argument ${ }^{15}$, we can assume that the number of phases is unbounded. The claim follows along the same line as the proof of Lemma 20 , replacing the notion of iteration with the notion of phase and the probability $1 / M$ with $p$.

We next bound the approximation factor of the algorithm for a generic sampling procedure (satisfying some properties).

\footnotetext{
$\overline{{ }^{14} \text { If } \mathbf{1}^{T} x=\frac{m}{N}}=O(1)$ and $|C|=O(1)$ for $C \in \mathbf{C}$, then the number of terminals would be bounded by a constant - in this case an optimum solution can be computed in polynomial time.

${ }^{15}$ The coupling works as follows. Let $\mathcal{A}$ be the original algorithm and $\mathcal{B}$ be the modified one (with an unbounded number of phases). We run the two algorithms with the same input sequence $\mathcal{I}$ of random bits. For any edge $e$, if $e$ is marked by $\mathcal{B}$ within a given phase $t$, then deterministically $e$ is marked by $\mathcal{A}$ as well within the same phase. Hence, $\operatorname{Pr}_{\mathcal{A}}[\bar{X}(\tilde{W}) \leq t] \geq \operatorname{Pr}_{\mathcal{B}}[\bar{X}(\tilde{W}) \leq t]$. We can conclude that $E_{\mathcal{A}}[\bar{X}(\tilde{W})]=\sum_{t \geq 1} \operatorname{Pr}_{\mathcal{A}}[\bar{X}(\tilde{W}) \geq t] \leq \sum_{t \geq 1} \operatorname{Pr}_{\mathcal{B}}[\bar{X}(\tilde{W}) \geq t]=E_{\mathcal{B}}[\bar{X}(\tilde{W})]$.

Journal of the ACM, Vol. ?, No. ?, ? 20?
} 
Lemma 24. Suppose that Step (1b) satisfies the following two properties:

(a) Each component $C$ is sampled with probability at most $\alpha \cdot x_{C}^{s}$

(b) Each edge $e$ in the witness tree is marked with probability at least $\beta$.

Then the approximation factor of the algorithm in Figure 5 is at most $\ln (4) \cdot\left(\frac{(1+\varepsilon) \alpha}{\beta}+\right.$ $\left.\frac{2 \varepsilon^{2}}{\beta}\right)$.

Proof. As in the proof of Theorem 21, one has

$$
\begin{gathered}
E[\bar{D}(e)] \quad=\quad \sum_{q=1}^{k_{e}} \operatorname{Pr}[|W(e)|=q] \cdot E[\bar{D}(e)|| W(e) \mid=q] \\
\stackrel{\text { Lem }}{\leq} \sum_{q \geq 1}^{18+23}\left(\frac{1}{2}\right)^{q} \cdot H_{q} \cdot \frac{1}{\beta}=\ln (4) \cdot \frac{1}{\beta} .
\end{gathered}
$$

Let $\mathrm{opt}^{s}$ be the cost of an optimal Steiner tree at the beginning of phase $s$. The expected cost of the sampled components satisfies

$$
\begin{aligned}
E\left[\sum_{s=1}^{1 / \varepsilon^{2}} \sum_{i=1}^{\phi_{s}} c\left(C^{s, i}\right)\right] & \leq \sum_{s=1}^{1 / \varepsilon^{2}} \sum_{C \in \mathbf{C}^{s}} E\left[\alpha \cdot x_{C}^{s} \cdot c(C)\right] \\
& \leq \alpha(1+\varepsilon) \cdot \sum_{s=1}^{1 / \varepsilon^{2}} E\left[\mathrm{opt}^{s}\right] \\
& \leq \alpha(1+\varepsilon) \sum_{e \in S^{*}} E[\bar{D}(e)] \cdot c(e) \stackrel{(2)}{\leq} \ln (4) \cdot \frac{\alpha(1+\varepsilon)}{\beta} \mathrm{opt} .
\end{aligned}
$$

Let $S^{\prime}:=\left\{e \in S^{*} \mid \bar{D}(e)>1 / \varepsilon^{2}\right\}$ be a feasible Steiner tree at the end of the last phase. By Markov's inequality and (2),

$$
\operatorname{Pr}\left[\bar{D}(e)>1 / \varepsilon^{2}\right] \leq \ln (4) \frac{\varepsilon^{2}}{\beta} .
$$

Therefore $E\left[c\left(S^{\prime}\right)\right] \leq \ln (4) \frac{\varepsilon^{2}}{\beta}$ opt. The minimum-cost terminal spanning tree is at most twice that expensive, hence $E[c(T)] \leq 2 \ln (4) \frac{\varepsilon^{2}}{\beta}$. opt. The claim follows.

Lemma 24 suggests an alternative way to implement the algorithm from Section 5. Consider the following natural implementation of Step (1b):

(Independent Phase Sampling)

Sample $\phi^{s}=\varepsilon \cdot M$ components $C^{s, 1}, \ldots, C^{s, \phi^{s}}$ independently (with repetitions), where $C^{s, i}=C \in \mathbf{C}^{s}$ with probability $x_{C}^{s} / M$.

The Independent Phase Sampling samples a component $C$ with a probability of at most $\phi^{s} \cdot \frac{1}{M} \cdot x_{C}^{s}=\varepsilon \cdot x_{C}^{s}$. On the other hand, the probability that edge $e \in W$ is marked is essentially lower bounded by $\varepsilon$. Inspecting Lemma 24, we see that $\alpha \approx \beta \approx \varepsilon$, which gives the following corollary.

COROLlary 25. The algorithm from Figure 5 which implements Step (1b) with the IndePendent Phase Sampling is $(\ln (4)+O(\varepsilon))$-approximate in expectation. 
This provides a 1.39-approximation algorithm that needs to solve just a constant (rather than polynomial) number of LPs. In particular, its running time might be competitive with the better-than-2 approximation algorithms in the literature. But a drawback of the IndePendent Phase Sampling implementation is that it needs too many (namely polynomially many) random bits: hence it is not easy to derandomize. For this reason we introduce a more complex sampling procedure in the next subsection.

\subsection{A Dependent Sampling Procedure}

We next describe an alternative implementation of Step (1b), which still guarantees $\alpha \approx \beta \approx \varepsilon$, and requires only $O(\log n)$ random bits. We focus on a specific phase $s$ and an edge $e \in W$. Let $(x, \mathbf{C}):=\left(x^{s}, \mathbf{C}^{s}\right)$. We renumber the components such that $\mathbf{C}=\left(C_{0}, \ldots, C_{m-1}\right)$, and we let $x_{j}:=x_{C_{j}}=\frac{1}{N}$.

(Dependent Phase Sampling)

(i) Choose $A \in\{0, \ldots, m-1\}$ and $B \in\{1, \ldots, m-1\}$ uniformly and independently at random.

(ii) Select $C_{j}$ with $j \in J:=\left\{A+i \cdot B \bmod m \mid i=1, \ldots,\left\lfloor\frac{\varepsilon}{N} m\right\rfloor\right\}$.

Observe that Step (i) requires only $O(\log m)$ random bits. Since $m=n^{O(1)}$, this number of bits is $O(\log n)$.

We will show that: (1) any component $C_{j}$ is sampled with probability no more than $\alpha x_{j}, \alpha:=\varepsilon$ and (2) edge $e$ is marked with probability at least $\beta:=\varepsilon(1-2 \varepsilon)$. The first claim is easy to show.

Lemma 26. Implementing Step (1b) with the Dependent Phase Sampling, each component $C_{j}$ is sampled with probability at most $\varepsilon \cdot x_{j}$.

Proof. For any component $C_{j}, \operatorname{Pr}[j \in J]=\frac{1}{m} \cdot\left\lfloor\frac{\varepsilon}{N} m\right\rfloor \leq \frac{\varepsilon}{N}=\varepsilon \cdot x_{j}$.

Showing claim (2) is more involved.

Lemma 27. Implementing Step (1b) with the Dependent Phase Sampling, each edge $e \in W$ is marked with probability at least $\varepsilon(1-2 \varepsilon)$.

Proof. Let $w_{C_{j}}$ be the probability distribution for component $C_{j}$ as in Lemma 19. Recall that $\operatorname{Pr}\left[\overline{\mathrm{Br}}_{W}\left(C_{j}\right)=B\right]=w_{C_{j}}(B)$ and

$$
\delta:=\sum_{j=0}^{m-1} x_{j} \sum_{B \in \mathcal{B}_{W}\left(C_{j}\right): e \in B} w_{C_{j}}(B) \geq 1 .
$$

Let $y_{j}:=\sum_{B \in \mathcal{B}_{W}\left(C_{j}\right): e \in B} w_{C_{j}}(B)$ denote the probability that $e$ is marked, given that $C_{j}$ is sampled. Since $x_{j}=\frac{1}{N}$, we have $\sum_{j=0}^{m-1} y_{j}=\delta N$. There lies no harm in assuming that $\delta=1$, since the probability that $e$ is marked is increasing in the $y_{j}$ 's.

Let $E_{j}$ be the event that $C_{j}$ is sampled and $e \in \overline{\mathrm{Br}}_{W}\left(C_{j}\right)$. It is sufficient to show that $\operatorname{Pr}\left[\bigcup_{j=0}^{m-1} E_{j}\right] \geq \varepsilon(1-2 \varepsilon)$. The crucial insight is to obtain a lower bound on $\operatorname{Pr}\left[E_{j}\right]$ and an upper bound on $\operatorname{Pr}\left[E_{j} \cap E_{j^{\prime}}\right]$ for $j \neq j^{\prime}$. First of all, we have

$$
\operatorname{Pr}\left[E_{j}\right]=y_{j} \cdot \operatorname{Pr}[j \in J]=y_{j} \cdot\left\lfloor\frac{\varepsilon m}{N}\right\rfloor \cdot \frac{1}{m} \geq \varepsilon(1-\varepsilon) \frac{y_{j}}{N},
$$

Journal of the ACM, Vol. ?, No. ?, ? 20?. 
using that $\frac{\varepsilon m}{N} \geq \frac{1}{\varepsilon}$ by assumption. Secondly, let $j, j^{\prime} \in\{0, \ldots, m-1\}$ be distinct component indices. Then $j, j^{\prime} \in J$ if and only if the system

$$
\begin{gathered}
j \equiv_{m} A+B i \\
j^{\prime} \equiv_{m} A+B i^{\prime}
\end{gathered}
$$

has a solution $i, i^{\prime}$. But since $\mathbb{Z}_{m}$ is a field, for any distinct pair $i, i^{\prime} \in\left\{1, \ldots,\left\lfloor\frac{\varepsilon m}{N}\right\rfloor\right\}$, there is precisely one pair $(A, B) \in\{0, \ldots, m-1\} \times\{1, \ldots, m-1\}$ satisfying (4). Hence

$$
\operatorname{Pr}\left[E_{j} \cap E_{j^{\prime}}\right] \leq y_{j} \cdot y_{j^{\prime}} \cdot\left\lfloor\frac{\varepsilon m}{N}\right\rfloor \cdot\left(\left\lfloor\frac{\varepsilon m}{N}\right\rfloor-1\right) \cdot \frac{1}{m \cdot(m-1)} \leq y_{j} \cdot y_{j^{\prime}} \cdot \frac{\varepsilon^{2}}{N^{2}} .
$$

By the inclusion-exclusion principle (see, e.g., Corollary A.2 in [Arora and Barak 2009]),

$$
\begin{aligned}
\operatorname{Pr}\left[\bigcup_{j=0}^{m-1} E_{j}\right] & \geq \sum_{j=0}^{m-1} \operatorname{Pr}\left[E_{j}\right]-\sum_{j=0}^{m-1} \sum_{j^{\prime} \neq j} \operatorname{Pr}\left[E_{j} \cap E_{j^{\prime}}\right] \\
& \geq \sum_{j=0}^{(3)+(5)} \varepsilon(1-\varepsilon) \frac{y_{j}}{N}-\frac{\varepsilon^{2}}{N^{2}} \underbrace{\sum_{j=0}^{m-1} y_{j}}_{=N} \cdot \underbrace{\sum_{j^{\prime} \neq j} y_{j^{\prime}}}_{\leq N} \\
& \geq \varepsilon(1-\varepsilon)-\varepsilon^{2}=\varepsilon(1-2 \varepsilon),
\end{aligned}
$$

which proves the claim.

A deterministic $(\ln (4)+\varepsilon)$-approximation algorithm easily follows.

Proof of Theorem 3. Consider the algorithm from Figure 5 which implements Step (1b) with the Dependent Phase Sampling. This algorithm can be derandomized by considering all the possible outcomes of random variables $A$ and $B$ in each phase, which are at most $m^{2 / \varepsilon^{2}}$. The claim on the approximation follows from Lemmas 24, 26, and 27 .

We can similarly derandomize the result for quasi-bipartite graphs.

TheOREM 28. For any constant $\varepsilon>0$, there is a deterministic polynomial-time algorithm for the Steiner tree problem on quasi-bipartite graphs with approximation ratio $\frac{73}{60}+\varepsilon$.

Proof. Consider the same algorithm as in Theorem 3. Lemmas 23, 26, and 27 still hold. Under the same assumptions as in Lemma 24, and by the different choice of the witness tree $W$ in this case, we now have

$$
E[\bar{D}(e)] \leq \frac{1}{k} \cdot H_{k-1} \cdot \frac{1}{\beta}+\left(1-\frac{1}{k}\right) \cdot H_{1} \cdot \frac{1}{\beta}=\left(\frac{1}{k} \cdot H_{k-1}+\frac{k-1}{k}\right) \cdot \frac{1}{\beta} \leq \frac{73}{60} \cdot \frac{1}{\beta} .
$$

Then the expected cost of the sampled components satisfies $E\left[\sum_{s, i} c\left(C^{s, i}\right)\right] \leq$ $\frac{73}{60} \frac{\alpha(1+\varepsilon)}{\beta}$ opt. Similarly, the expected cost of the final spanning tree satisfies $E[c(T)] \leq 2 \cdot \frac{73}{60} \frac{\varepsilon^{2}}{\beta} \cdot$ opt. Altogether, the approximation factor from Lemma 24 now reduces to $\frac{73}{60} \cdot\left(\frac{(1+\varepsilon) \alpha}{\beta}+\frac{2 \varepsilon^{2}}{\beta}\right)$. The claim follows along the same line as in Theorem 3. 


\section{INTEGRALITY GAP}

In this section we upper bound (Section 7.1) and lower bound (Section 7.2) the integrality gap of DCR. Furthermore, we compare DCR with BCR (Section 7.3).

\subsection{An Upper Bound}

Note that, despite the fact that our analysis is based on an LP relaxation of the problem, it does not imply a $\ln (4)$ (nor even a 1.5) upper bound on the integrality gap of the studied LP. It only provides a $1+\ln (2)$ upper bound, as shown in Theorem 13 (by letting $\varepsilon$ tend to zero). This is because the LP changes during the iterations of the algorithm, and its solution is only bounded with respect to the initial optimal integral solution. In this section we prove that our LP has integrality gap at most $1+\ln (3) / 2<1.55$. Before proceeding with our (fairly technical) argument, let us remark that, after the conference version of this paper appeared, a shorter and perhaps more elegant proof (still based on the Bridge Lemma) of the same claim was given in [Chakrabarty et al. 2010b].

In order to prove the 1.55 upper bound on the integrality gap of DCR, claimed in Theorem 4, we consider the algorithm R\&Z by Robins and Zelikovsky [2005]. We show that this algorithm produces solutions of cost bounded with respect to the optimal fractional solutions to $k$-DCR (and hence of DCR). This is achieved by combining the original analysis in [Robins and Zelikovsky 2005] with our new Bridge Lemma 11. This approach was, to some extent, inspired by an argument in [Charikar and Guha 2005] in the context of facility location. We leave it as an interesting open problem to prove a $\ln (4$ ) (or even 1.5) upper bound on the integrality gap of DCR (if possible). This might involve the development of a fractional version of Lemma 14 .

Algorithm R\&Z works as follows. It constructs a sequence $T^{0}, T^{1}, \ldots, T^{\mu}$ of terminal spanning trees, where $T^{0}$ is a minimum-cost terminal spanning tree in the original graph. At iteration $t$ we are given a tree $T^{t}$ and a cost function $c^{t}$ on the edges of the tree (initially $c^{0} \equiv c$ ). The algorithm considers any candidate component $C$ with at least 2 and at most $k$ terminals (k-component). Let $T^{t}[C]$ denote the minimum spanning tree of the graph $T^{t} \cup C$, where the edges $e \in C$ have weight 0 and the edges $f \in T^{t}$ weight $c^{t}(f)$. The subset of edges in $T^{t}$ but not in $T^{t}[C]$ are denoted by $\mathrm{Br}_{T^{t}}(C)$. In fact, $\mathrm{Br}_{T^{t}}(C)$ is the set of bridges of $T^{t}$ with respect to $R(C)$ and the above weight function. For a given component $C$, we denote as $\operatorname{Loss}(C)$ the minimum-cost subforest of $C$ with the property that there is a path between each Steiner node in $C$ and some terminal in $R(C)$. In the terminology from Section 3, $\operatorname{Loss}(C)$ is the complement of the set of bridges of the subtree $C$ after contracting $R(C)$. We let $\operatorname{loss}(C)=c(\operatorname{Loss}(C))$.

It is convenient to define the following quantities:

$$
\operatorname{gain}^{t}(C)=\operatorname{br}_{T^{t}}(C)-c(C) \quad \text { and } \quad \operatorname{sgain}^{t}(C)=\operatorname{gain}^{t}(C)+\operatorname{loss}(C) .
$$

The algorithm selects the component $C^{t+1}$ which maximizes gain ${ }^{t}(C) / \operatorname{loss}(C)$. If this quantity is non-positive, the algorithm halts. Otherwise, it considers the graph $T^{t} \cup C^{t+1}$, and contracts $\operatorname{Loss}\left(C^{t+1}\right)$. The tree $T^{t+1}$ is a minimum-cost terminal spanning tree in the resulting graph. In case that parallel edges are created this way, the algorithm only keeps the cheapest of such edges. This way we obtain the

Journal of the ACM, Vol. ?, No. ?, ? 20?. 
cost function $c^{t+1}$ on the edges of $T^{t+1}$.

Let $\mathrm{Apx}_{k}$ be the approximate solution computed by the algorithm, and apx ${ }_{k}=$ $c\left(\mathrm{Apx}_{k}\right)$. Recall that $\mu$ is the index of the final tree in the sequence of computed trees. The following two lemmas are implicitly given in [Robins and Zelikovsky 2005]: the first one is implied by Equation (4.2), and the second one is contained in the first chain of inequalities at page 8 .

Lemma 29. [Robins and Zelikovsky 2005] Fort $=1,2, \ldots, \mu, c^{t}\left(T^{t}\right)=c^{t-1}\left(T^{t-1}\right)-$ $\operatorname{sgain}^{t-1}\left(C^{t}\right)$.

Lemma 30. [Robins and Zelikovsky 2005] For any $\ell \leq \mu$,

$$
\operatorname{apx}_{k} \leq \sum_{t=1}^{\ell} \operatorname{loss}\left(C^{t}\right)+c^{\ell}\left(T^{\ell}\right)
$$

Recall that opt $f, k$ is the cost of the optimal fractional solution to $k$-DCR. Let $(x, \mathbf{C})$ be an optimal fractional solution to $k$-DCR. Define $\operatorname{loss}_{f, k}:=\sum_{C \in \mathbf{C}} x_{C} \operatorname{loss}(C)$.

Corollary $31 \cdot \operatorname{loss}_{f, k} \leq \frac{1}{2} \operatorname{opt}_{f, k}$.

Proof. From Lemma 6, for any $C \in \mathbf{C}$, $\operatorname{loss}(C)=c(C)-\operatorname{br}_{C}(R(C)) \leq \frac{1}{2} c(C)$. As a consequence, $\operatorname{loss}_{f, k} \leq \frac{1}{2} \sum_{C \in \mathbf{C}} x_{C} \cdot c(C)=\frac{1}{2} \operatorname{opt}_{f, k}$.

Corollary 32. $c^{\mu}\left(T^{\mu}\right) \leq$ opt $_{f, k}$.

Proof. Using the fact that gain ${ }^{\mu}(C)=\mathrm{br}_{T^{\mu}}(C)-c(C) \leq 0$ for any component $C$,

$$
c^{\mu}\left(T^{\mu}\right) \stackrel{\text { Bridge Lem } 11}{\leq} \sum_{C \in \mathbf{C}} x_{C} \operatorname{br}_{T^{\mu}}(C) \leq \sum_{C \in \mathbf{C}} x_{C} c(C)=\operatorname{opt}_{f, k} .
$$

By Corollary 32, and since $c^{t}\left(T^{t}\right)$ is a non-increasing function of $t$, there must be a value of $\ell \leq \mu$ such that:

$$
c^{\ell-1}\left(T^{\ell-1}\right)>\operatorname{opt}_{f, k} \geq c^{\ell}\left(T^{\ell}\right)
$$

In the following we will bound $\sum_{t=1}^{\ell} \operatorname{loss}\left(C^{t}\right)+c^{\ell}\left(T^{\ell}\right)$. By Lemma 30, this will give a bound on $\operatorname{apx}_{k}$. Let

$$
\operatorname{gain}_{f}^{t}:=c^{t}\left(T^{t}\right)-\operatorname{opt}_{f, k} \quad \text { and } \quad \operatorname{sgain}_{f}^{t}:=\operatorname{gain}_{f}^{t}+\operatorname{loss}_{f, k} .
$$

Lemma 33. For $t=1,2, \ldots, \mu, \frac{\operatorname{sgain}^{t-1}\left(C^{t}\right)}{\operatorname{loss}\left(C^{t}\right)} \geq \frac{\operatorname{sgain}_{f}^{t-1}}{\operatorname{loss}_{f, k}}$. 
Proof. We first note that

$$
\begin{aligned}
\frac{\operatorname{gain}_{f}^{t-1}}{\operatorname{loss}_{f, k}} & =\frac{c^{t-1}\left(T^{t-1}\right)-\sum_{C \in \mathbf{C}} x_{C} c(C)}{\sum_{C \in \mathbf{C}} x_{C} \operatorname{loss}(C)} \\
& \leq \underset{\text { Bridge Lem } 11}{\leq} \frac{\sum_{C \in \mathbf{C}} x_{C}\left(\operatorname{br}_{T^{t-1}}(C)-c(C)\right)}{\sum_{C \in \mathbf{C}} x_{C} \operatorname{loss}(C)} \\
& =\frac{\sum_{C \in \mathbf{C}} x_{C} \operatorname{gain}^{t-1}(C)}{\sum_{C \in \mathbf{C}} x_{C} \operatorname{loss}(C)} \\
& \leq \max _{C \in \mathbf{C}}\left\{\frac{\operatorname{gain}^{t-1}(C)}{\operatorname{loss}(C)}\right\} \leq \frac{\operatorname{gain}^{t-1}\left(C^{t}\right)}{\operatorname{loss}\left(C^{t}\right)},
\end{aligned}
$$

where in the last inequality we used the fact that $C^{t}$ maximizes gain ${ }^{t-1}(C) / \operatorname{loss}(C)$ over all the $k$-restricted components $C$. It follows that

$$
\frac{\operatorname{sgain}^{t-1}\left(C^{t}\right)}{\operatorname{loss}\left(C^{t}\right)}=1+\frac{\operatorname{gain}^{t-1}\left(C^{t}\right)}{\operatorname{loss}\left(C^{t}\right)} \geq 1+\frac{\operatorname{gain}_{f}^{t-1}}{\operatorname{loss}_{f, k}}=\frac{\operatorname{sgain}_{f}^{t-1}}{\operatorname{loss}_{f, k}} .
$$

We need some more notation. Let $\operatorname{sgain}^{\ell-1}\left(C^{\ell}\right)=\operatorname{sgain}^{1}+\operatorname{sgain}^{2}$ such that

$$
\operatorname{sgain}^{1}=c^{\ell-1}\left(T^{\ell-1}\right)-\operatorname{opt}_{f, k} \stackrel{(6)}{>} 0 .
$$

We also let $\operatorname{loss}\left(C^{\ell}\right)=\operatorname{loss}^{1}+\operatorname{loss}^{2}$ such that

$$
\frac{\operatorname{sgain}^{\ell-1}\left(C^{\ell}\right)}{\operatorname{loss}\left(C^{\ell}\right)}=\frac{\operatorname{sgain}^{1}}{\operatorname{loss}^{1}}=\frac{\operatorname{sgain}^{2}}{\operatorname{loss}^{2}} .
$$

Eventually, we define

$$
\begin{aligned}
\operatorname{sgain}_{f}^{\ell 1} & :=\operatorname{sgain}_{f}^{\ell-1}-\operatorname{sgain}^{1} \\
& \stackrel{(7)}{=} c^{\ell-1}\left(T^{\ell-1}\right)-\operatorname{opt}_{f, k}+\operatorname{loss}_{f, k}-\left(c^{\ell-1}\left(T^{\ell-1}\right)-\operatorname{opt}_{f, k}\right)=\operatorname{loss}_{f, k} .
\end{aligned}
$$

Lemma 34. $\sum_{t=1}^{\ell-1} \operatorname{loss}\left(C^{t}\right)+\operatorname{loss}^{1} \leq \operatorname{loss}_{f, k} \ln \left(\frac{\operatorname{sgain}_{f}^{0}}{\operatorname{sgain}_{f}^{\ell_{1}^{1}}}\right)$.

Proof. For every $t=1,2, \ldots, \ell-1$,

$$
\operatorname{sgain}_{f}^{t}=\operatorname{sgain}_{f}^{t-1}-\operatorname{sgain}^{t-1}\left(C^{t}\right) \stackrel{\operatorname{Lem}}{\leq} 33 \operatorname{sgain}_{f}^{t-1}\left(1-\frac{\operatorname{loss}\left(C^{t}\right)}{\operatorname{loss}_{f, k}}\right) .
$$

Furthermore

from which

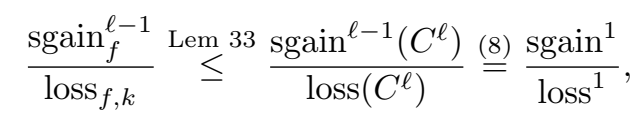

$$
\operatorname{sgain}_{f}^{\ell 1}=\operatorname{sgain}_{f}^{\ell-1}-\operatorname{sgain}^{1} \leq \operatorname{sgain}_{f}^{\ell-1}\left(1-\frac{\operatorname{loss}^{1}}{\operatorname{loss}_{f, k}}\right) .
$$

Then

$$
\frac{\operatorname{sgain}_{f}^{\ell 1}}{\operatorname{sgain}_{f}^{0}} \leq\left(1-\frac{\operatorname{loss}^{1}}{\operatorname{loss}_{f, k}}\right) \prod_{t=1}^{\ell-1}\left(1-\frac{\operatorname{loss}\left(C^{t}\right)}{\operatorname{loss}_{f, k}}\right) .
$$

Journal of the ACM, Vol. ?, No. ?, ? 20?. 
Taking the logarithm of both sides and recalling that $x \geq \ln (1+x)$,

$$
\ln \left(\frac{\operatorname{sgain}_{f}^{0}}{\operatorname{sgain}_{f}^{\ell 1}}\right) \geq \frac{1}{\operatorname{loss}_{f, k}}\left(\sum_{t=1}^{\ell-1} \operatorname{loss}\left(C^{t}\right)+\operatorname{loss}^{1}\right) .
$$

We now have all the ingredients to bound the approximation factor of the algorithm with respect to opt $f, k$. Let mst $=c\left(T^{0}\right)=c^{0}\left(T^{0}\right)$. The following theorem and corollary are straightforward adaptations of analogous results in [Robins and Zelikovsky 2005].

THEOREM 35. $\operatorname{apx}_{k} \leq \mathrm{opt}_{f, k}+\operatorname{loss}_{f, k} \ln \left(\frac{\mathrm{mst}_{-\mathrm{opt}_{f, k}+\operatorname{loss}_{f, k}}}{\operatorname{loss}_{f, k}}\right)$.

Proof. Since $\operatorname{sgain}^{t-1}\left(C^{t}\right) \geq \operatorname{loss}\left(C^{t}\right)$, it follows from (8) that

$$
\operatorname{sgain}^{2} \geq \operatorname{loss}^{2}
$$

Putting everything together we obtain

$$
\begin{aligned}
\operatorname{apx}_{k} & \stackrel{\text { Lem }}{\leq} \sum_{t=1}^{\ell 0} \operatorname{loss}\left(C^{t}\right)+c^{\ell}\left(T^{\ell}\right) \\
& \stackrel{\text { Lem }}{=} 29 \sum_{t=1}^{\ell-1} \operatorname{loss}\left(C^{t}\right)+\operatorname{loss}\left(C^{\ell}\right)+c^{\ell-1}\left(T^{\ell-1}\right)-\operatorname{sgain}^{\ell-1}\left(C^{\ell}\right) \\
& =\sum_{t=1}^{\ell-1} \operatorname{loss}\left(C^{t}\right)+\operatorname{loss}^{1}+\operatorname{loss}^{2}+c^{\ell-1}\left(T^{\ell-1}\right)-\operatorname{sgain}^{1}-\operatorname{sgain}^{2} \\
& \stackrel{(10)}{\leq} \sum_{t=1}^{\ell-1} \operatorname{loss}\left(C^{t}\right)+\operatorname{loss}^{1}+c^{\ell-1}\left(T^{\ell-1}\right)-\operatorname{sgain}^{1} \\
& \stackrel{(7)}{=} \sum_{t=1}^{\ell-1} \operatorname{loss}\left(C^{t}\right)+\operatorname{loss}^{1}+\operatorname{opt}_{f, k} \\
& \stackrel{\text { Lem } 34}{\leq} \operatorname{opt}_{f, k}+\operatorname{loss}_{f, k} \ln \left(\frac{\operatorname{sgain}_{f}^{0}}{\operatorname{sgain}_{f}^{\ell 1}}\right) \\
& \stackrel{(9)}{=} \operatorname{opt}_{f, k}+\operatorname{loss}_{f, k} \ln \left(\frac{\operatorname{mst}^{\ell} \operatorname{opt}_{f, k}+\operatorname{loss}_{f, k}}{\operatorname{loss}_{f, k}}\right) .
\end{aligned}
$$

Lemma 36. For any constant $k \geq 2$, there exists a polynomial-time algorithm for Steiner tree which computes a solution of cost at most $1+\ln (3) / 2$ times the cost of the optimal fractional solution to $k$-DCR.

Proof. A straightforward adaptation of Lemma 5 implies that

$$
\text { mst } \leq 2 \operatorname{opt}_{f, k} \text {. }
$$

Combining the inequality above with Theorem 35 , we obtain

$$
\begin{aligned}
& \operatorname{apx}_{k} \leq \operatorname{opt}_{f, k}+\operatorname{loss}_{f, k} \ln \left(1+\frac{2 \mathrm{opt}_{f, k}-\mathrm{opt}_{f, k}}{\operatorname{loss}_{f, k}}\right) . \\
& \text { Journal of the ACM, Vol. ?, No. ?, ? 20?. }
\end{aligned}
$$




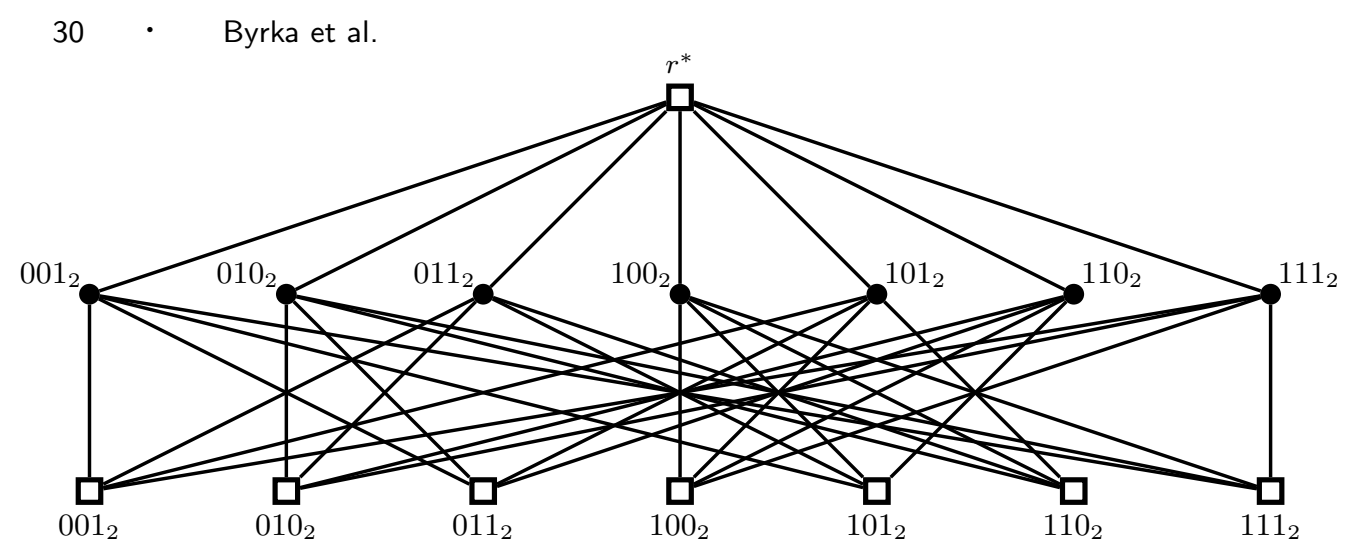

Fig. 6. Skutella's graph. Nodes are labeled with their indices in binary representation.

The right-hand side of the inequality above is an increasing function of $\operatorname{loss}_{f, k}$. By Corollary $31, \operatorname{loss}_{f, k} \leq \frac{1}{2} \operatorname{opt}_{f, k}$, which implies

$$
\operatorname{apx}_{k} \leq \operatorname{opt}_{f, k}+\frac{1}{2} \mathrm{opt}_{f, k} \ln \left(1+2 \frac{2 \mathrm{opt}_{f, k}-\mathrm{opt}_{f, k}}{\mathrm{opt}_{f, k}}\right)=\operatorname{opt}_{f, k}\left(1+\frac{\ln (3)}{2}\right) .
$$

Theorem 4 follows.

Proof of Theorem 4. From Lemma $7, \mathrm{opt}_{f, k} \leq \rho_{k} \cdot \mathrm{opt}_{f}$. The claim follows from Lemma 36 and Theorem 1 by choosing a large-enough $k$.

\subsection{A Lower Bound}

The best-known lower bound on the integrality gap of BCR (prior to our work) is achieved by a family of instances due to Goemans, whose gap tends to $8 / 7$, as well as a single instance due to Skutella with a gap of precisely $8 / 7$ (see [Könemann et al. 2011]).

\section{THEOREM 37. The integrality gap of DCR is at least 8/7 $>1.142$.}

Proof. We will use Skutella's graph [Könemann et al. 2011]. Consider a Set Cover instance with elements $U=\{1, \ldots, 7\}$ and sets $S_{1}, \ldots, S_{7}$. Let $b(i)$ be a vector from $\mathbb{Z}_{2}^{3}$ that is the binary representation of $i$, for example $b(3)=(0,1,1)$. We define the sets by $S_{j}:=\left\{i \in U \mid b(i) \cdot b(j) \equiv_{2} 1\right\}$. Note that this is exactly the definition of the instance which yields a $\Omega(\log n)$ lower bound on the integrality gap of Set Cover for $n=7$ [Vazirani 2001]. The critical property is that for our particular instance one needs 3 sets to cover all elements, but choosing each set to an extent of $1 / 4$ gives a fractional Set Cover solution of cost $7 / 4$.

Next we define a graph where each element forms a terminal and each set is a non-terminal node connected to the root and to the contained elements by unit cost edges (see Figure 6).

If we direct all the edges upwards, the graph can be decomposed into 7 edgedisjoint components, each one containing one non-terminal node and the 5 edges incident into it. On one hand installing $1 / 4$ on each of these components gives a fractional solution of cost 35/4, while on the other hand at least 3 Steiner nodes

Journal of the ACM, Vol. ?, No. ?, ? 20?. 


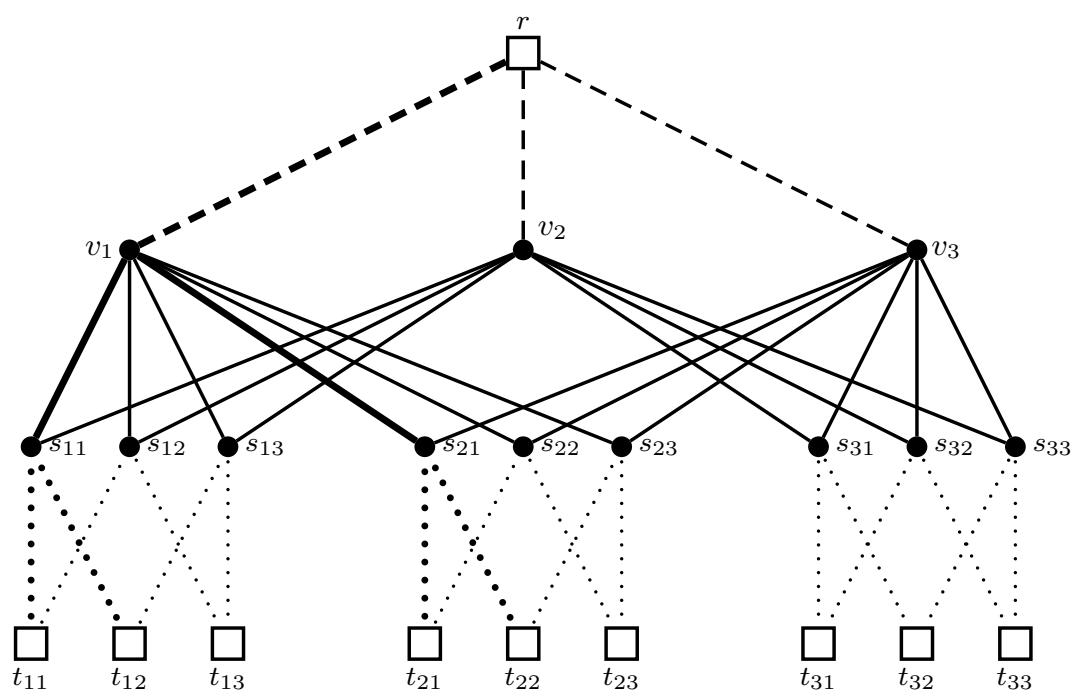

Fig. 7. All edges have cost 1 . The unique optimal solution to BCR, of cost 15, installs capacity $1 / 4$ on the central (full) edges and capacity $1 / 2$ on the remaining (dashed and dotted) edges, always directed upwards. The cheapest solution to DCR has cost $7 / 4 \cdot 9=15.75$ (one component is drawn in bold; install $\frac{1}{12}$ capacity units on all $3^{3}$ symmetric components. Each terminal lies in $3^{3} \cdot\left(\frac{2}{3}\right)^{2}=12$ such components, thus the solution is feasible. In fact, it must be optimal since every possible component has $\frac{\# \text { connected terminals }}{\text { \#edges }} \geq \frac{7}{4}$ as one can easily check). The overall capacity reserved on each edge is the same as in the BCR case, excluding the top (dashed) edges, where the capacity is $3 / 4$. The (integral) optimal Steiner tree has cost 17 .

must be included for an integer solution. Consequently opt $=10$ and we obtain the promised gap of $\frac{10}{35 / 4}=\frac{8}{7}$.

\subsection{Comparison with $\mathrm{BCR}$}

We start by observing that DCR is a relaxation strictly stronger than BCR.

LEMMA 38. Let opt $_{D C R}$ and $\mathrm{opt}_{B C R}$ be the optimal fractional solutions to DCR and $B C R$, respectively, for a given input instance. Then $\operatorname{opt}_{D C R} \geq \mathrm{opt}_{B C R}$ and there are examples where strict inequality holds.

Proof. Any feasible solution to DCR can be turned into a feasible solution to $\mathrm{BCR}$ of the same cost. In fact, it is sufficient to split each component into the corresponding set of edges. This proves the first part of the claim. An example of strict inequality is given in Figure 7.

Observe that the 1.55 upper bound on the integrality gap of DCR does not imply the same bound on the integrality gap of BCR. It remains as a challenging open problem to show whether the integrality gap of BCR is smaller than 2 or not.

The best-known lower bound on the integrality gap of BCR is 8/7 > 1.142 [Könemann et al. 2011; Vazirani 2001]. In particular, the family of instances which provides this bound is the same as in Section 7.2. We next present an improved lower bound of $36 / 31$ on the integrality gap of BCR. 


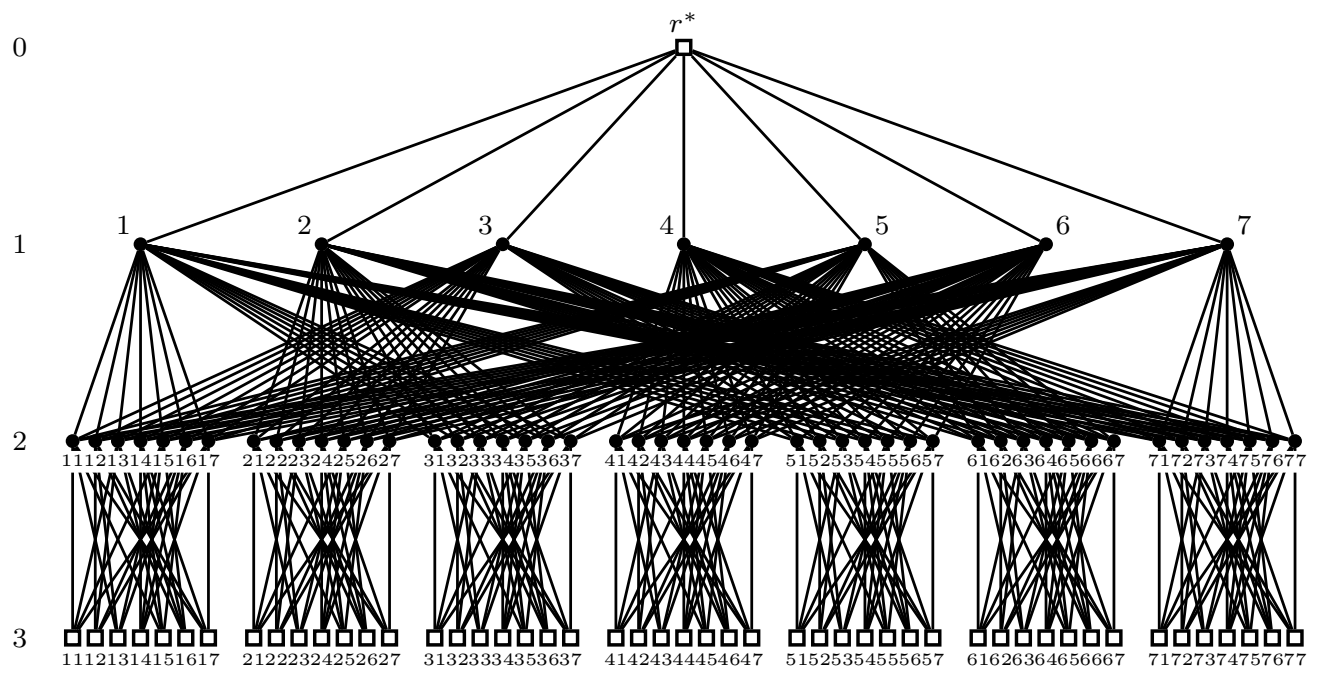

Fig. 8. Instance for $p=2$. Nodes are labeled with the corresponding vector in abbreviated notation; all edges have unit costs. The optimal fractional solution consist of installing capacity $1 / 16$ on each edge from level 2 to level 1 and capacity $1 / 4$ otherwise (always directed "upwards"), thus opt $f=7^{2}+7^{2} / 4+7 / 4=63$. On the other hand for an integer solution one needs $3+3 \cdot 7+7^{2}=$ 73 edges. The gap for this instance is consequently $\frac{73}{63} \approx 1.158$.

\section{TheOREM 39. The integrality gap of BCR is at least $36 / 31>1.161$.}

Proof. The basic idea is generalizing the construction used in Section 7.2. Let $p \in \mathbb{N}$ be a parameter. We create a graph with $p+2$ levels and unit cost edges. For $i \in\{1, \ldots, p\}$ one has $7^{i}$ non-terminal nodes on the $i$ th level, each represented by a vector from $U^{i}$, where $U=\{1, \ldots, 7\}$. Furthermore we have a root terminal on level 0 and $7^{p}$ terminals on the $(p+1)$ th level, represented by vectors from $U^{p}$. We connect the root to all nodes in the first level. For $i=1, \ldots, p$, consider nodes $u=\left(u_{1}, \ldots, u_{i}\right) \in U^{i}$ on level $i$ and $v=\left(v_{1}, \ldots, v_{i+1}\right) \in U^{i+1}$ on level $i+1$. We connect $u$ and $v$ by an edge if $\left(u_{1}, \ldots, u_{i-1}\right)=\left(v_{1}, \ldots, v_{i-1}\right)$ and $b\left(u_{i}\right) \cdot b\left(v_{i}\right) \equiv_{2} 1$. We connect the non-terminal node $u \in U^{p}$ on level $p$ with terminal $v \in U^{p}$ on level $p+1$ in a similar manner, namely if and only if $\left(u_{1}, \ldots, u_{p-1}\right)=\left(v_{1}, \ldots, v_{p-1}\right)$ and $b\left(u_{p}\right) \cdot b\left(v_{p}\right) \equiv_{2} 1$. Observe that, for $p=1$, we obtain exactly Skutella's graph. The graph obtained for $p=2$ is depicted in Figure 8.

Let us consider any integer optimal solution, of cost opt, and direct the edges towards $r^{*}$. Each time we have an edge going from a level $i$ downwards to level $i+1$ we can replace it by an edge to level $i-1$ without disconnecting the tree. Observe that, for $i=0, \ldots, p-1$, we need at least $3 \cdot 7^{i}$ edges between level $i$ and $i+1$ and that $7^{p}$ edges are needed between the last two levels. This amount of edges is also sufficient, thus

$$
\text { opt }=3 \cdot\left(7^{0}+7^{1}+\ldots+7^{p-1}\right)+7^{p}=\frac{3}{2} \cdot 7^{p}-\frac{1}{2} .
$$

Consider now the optimal fractional solution to BCR for the same instance. Let $\mathrm{opt}_{f}^{p}$ denote its cost. This solution installs capacity $1 / 4$ on the edges incident to the

Journal of the ACM, Vol. ?, No. ?, ? 20?. 
root and to the terminals, and capacity $1 / 16$ on the remaining edges (all directed upwards). Hence

$$
\mathrm{opt}_{f}^{p}=\frac{4}{4} 7^{p}+\frac{4}{16} \cdot\left(7^{1}+7^{2}+\ldots+7^{p}\right)=\frac{31}{24} \cdot 7^{p}-\frac{7}{24} .
$$

The claim follows since

$$
\lim _{p \rightarrow \infty} \frac{\mathrm{opt}^{p}}{\mathrm{opt}_{f}^{p}}=\frac{36}{31} .
$$

\section{ACKNOWLEDGMENTS}

The authors wish to thank C. Chekuri, F. Eisenbrand, M. X. Goemans, J. Könemann, D. Pritchard, F. B. Shepherd, and R. Zenklusen for helpful discussions. The second author is grateful to F. Eisenbrand for supporting his visit at EPFL (during which part of this paper was developed). We thank the anonymous reviewers of the conference and journal version of this paper for many suggestions on how to improve the presentation.

\section{REFERENCES}

Agrawal, A., Klein, P., and Ravi, R. 1995. When trees collide: An approximation algorithm for the generalized Steiner problem on networks. SIAM Journal on Computing 24, 440-456.

Alon, N. And Spencer, J. 2008. The probabilistic method. John Wiley \& Sons Inc.

Archer, A., Bateni, M., Hajiaghayi, M., and Karloff, H. 2011. Improved approximation algorithms for prize-collecting Steiner tree and TSP. SIAM Journal on Computing, 40, 2, 309-332.

Arora, S. 1998. Polynomial time approximation schemes for Euclidean traveling salesman and other geometric problems. Journal of the ACM 45, 5, 753-782.

Arora, S. AND BARAK, B. 2009. Computational complexity: A modern approach. Cambridge University Press, Cambridge.

Bern, M. and Plassmann, P. 1989. The Steiner problem with edge lengths 1 and 2. Information Processing Letters 32, 4, 171-176.

Borchers, A. And Du, D. 1997. The $k$-Steiner ratio in graphs. SIAM Journal on Computing 26, 3, 857-869.

Borradaile, G., Klein, P., And Mathieu, C. 2009. An $O(n \log n)$ approximation scheme for Steiner tree in planar graphs. ACM Transactions on Algorithms 5, 3.

Byrka, J., Grandoni, F., Rothvoss, T., And Sanità, L. 2010. An improved LP-based approximation for Steiner tree. In ACM Symposium on Theory of Computing (STOC). 583-592.

Chakrabarty, D., Devanur, N. R., And Vazirani, V. V. 2008. New geometry-inspired relaxations and algorithms for the metric Steiner tree problem. In International Conference on Integer Programming and Combinatorial Optimization (IPCO). 344-358.

Chakrabarty, D., Könemann, J., and Pritchard, D. 2010a. Hypergraphic LP relaxations for Steiner trees. In International Conference on Integer Programming and Combinatorial Optimization (IPCO). 383-396.

Chakrabarty, D., Könemann, J., And Pritchard, D. 2010b. Integrality gap of the hypergraphic relaxation of Steiner trees: a short proof of a 1.55 upper bound. Operations Research Letters 38(6), 567-570.

Charikar, M. And GuHA, S. 2005. Improved combinatorial algorithms for facility location problems. SIAM Journal on Computing 34, 803-824.

Chlebík, M. And ChlebíkovÁ, J. 2008. The Steiner tree problem on graphs: Inapproximability results. Theoretical Computer Science 406, 3, 207-214.

Journal of the ACM, Vol. ?, No. ?, ? 20?. 
Dreyfus, S. E. And Wagner, R. A. 1972. The Steiner problem in graphs. Networks 1, 195-207.

Edmonds, J. 1967. Optimum branchings. Journal of Research of the National Bureau of Standards B71, 233-240.

Eisenbrand, F. AND Grandoni, F. 2005. An improved approximation algorithm for virtual private network design. In ACM-SIAM Symposium on Discrete Algorithms (SODA). 928-932.

Eisenbrand, F., Grandoni, F., Oriolo, G., and Skutella, M. 2007. New approaches for virtual private network design. SIAM Journal on Computing 37, 3, 706-721.

Eisenbrand, F., Grandoni, F., Rothvoss, T., And Schäfer, G. 2010. Connected facility location via random facility sampling and core detouring. Journal of Computer and System Sciences 76, 8, 709-726.

Frank, A. AND TARdos, É. 1987. An application of simultaneous Diophantine approximation in combinatorial optimization. Combinatorica 7, 49-65.

Garey, M. R. and Johnson, D. S. 1979. Computers and intractability. W. H. Freeman and Co.

Gilbert, E. N. And Pollak, H. O. 1968. Steiner minimal trees. SIAM Journal on Applied Mathematics 16, 1, 1-29.

Goemans, M. X. And Myung, Y. 1993. A catalog of Steiner tree formulations. Networks 23, 1, $19-28$.

Goemans, M. X. And Williamson, D. 1995. A general approximation technique for constrained forest problems. SIAM Journal on Computing 24, 296-317.

Grandoni, F. And Italiano, G. F. 2006. Improved approximation for single-sink buy-at-bulk. In International Symposium on Algorithms and Computation (ISAAC). 111-120.

Grandoni, F. And Rothvoss, T. 2011. Approximation algorithms for single and multi-commodity connected facility location. In International Conference on Integer Programming and Combinatorial Optimization (IPCO). 248-260.

Grandoni, F., Rothvoss, T., and Sanità, L. 2011. From uncertainty to nonlinearity: Solving virtual private network via single-sink buy-at-bulk. Mathematics of Operations Research 36, 2, $185-204$.

Gröpl, C., Hougardy, S., Nierhoff, T., And Prömel, H.-J. 2002. Steiner trees in uniformly quasi-bipartite graphs. Information Processing Letters 83, 4, 195-200.

Grötschel, M., Lovasz, L., And Schrijver, A. 1981. The ellipsoid method and its consequences in combinatorial optimization. Combinatorica 1, 169-197.

Gupta, A., Kleinberg, J., Kumar, A., Rastogi, R., and Yener, B. 2001. Provisioning a virtual private network: a network design problem for multicommodity flow. In ACM Symposium on Theory of Computing (STOC). 389-398.

Gupta, A., Kumar, A., PÁl, M., And Roughgarden, T. 2007. Approximation via cost sharing: Simpler and better approximation algorithms for network design. Journal of the ACM 54, 3, 11.

Hwang, F., Richards, D., And Winter, P. 1992. The Steiner tree problem. Monograph in Annals of Discrete Mathematics, 53. Elsevier.

JAIN, K. 2001. A factor 2 approximation algorithm for the generalized Steiner network problem. Combinatorica 21, 1, 39-60.

Jothi, R. AND RAGHAVACHARI, B. 2009. Improved approximation algorithms for the single-sink buy-at-bulk network design problems. Journal of Discrete Algorithms 7, 2, 249-255.

Karpinski, M. And Zelikovsky, A. 1997. New approximation algorithms for the Steiner tree problem. Journal of Combinatorial Optimization 1, 1, 47-65.

Khachiyan, L. G. 1979. A polynomial algorithm for linear programming. Soviet Mathematics Doklady 20, 191-194. (Russian original in Doklady Akademiia Nauk SSSR, 244:1093-1096).

Könemann, J., Pritchard, D., And TAn, K. 2011. A partition-based relaxation for Steiner trees. Mathematical Programming 127, 2, 345-370.

Mitzenmacher, M. And Upfal, E. 2005. Probability and computing. Cambridge University Press, Cambridge. Randomized algorithms and probabilistic analysis.

Journal of the ACM, Vol. ?, No. ?, ? 20? 
Mölle, D., Richter, S., And Rossmanith, P. 2006. A faster algorithm for the Steiner tree problem. In Annual Symposium on Theoretical Aspects of Computer Science (STACS). 561570 .

Niven, I., Zuckerman, H. S., And Montgomery, H. L. 1991. An introduction to the theory of numbers. John Wiley \& Sons Inc.

Polzin, T. And Vahdati-Daneshmand, S. 2003. On Steiner trees and minimum spanning trees in hypergraphs. Operations Research Letters 31, 1, 12-20.

Prömel, H. J. And Steger, A. 2000. A new approximation algorithm for the Steiner tree problem with performance ratio 5/3. Journal of Algorithms 36, 89-101.

RAJAgOPAlan, S. AND VAzIRANi, V. V. 1999. On the bidirected cut relaxation for the metric Steiner tree problem. In ACM-SIAM Symposium on Discrete Algorithms (SODA). 742-751.

Rizzi, R. 2003. On Rajagopalan and Vazirani's 3/2-approximation bound for the Iterated 1Steiner heuristic. Information Processing Letters 86, 6, 335-338.

Robins, G. And Zelikovsky, A. 2000. Improved Steiner tree approximation in graphs. In $A C M$ SIAM Symposium on Discrete Algorithms (SODA). 770-779.

Robins, G. And Zelikovsky, A. 2005. Tighter bounds for graph Steiner tree approximation. SIAM Journal on Discrete Mathematics 19, 1, 122-134.

Swamy, C. And Kumar, A. 2004. Primal-dual algorithms for connected facility location problems. Algorithmica 40, 4, 245-269.

TALWAR, K. 2002. The single-sink buy-at-bulk LP has constant integrality gap. In International Conference on Integer Programming and Combinatorial Optimization (IPCO). 475-486.

Vazirani, V. V. 2001. Approximation Algorithms. Springer-Verlag.

Zelikovsky, A. 1993. An 11/6-approximation algorithm for the network Steiner problem. Algorithmica 9, 463-470. 